\title{
Nakao's method and an interval difference scheme of second order for solving the elliptic BVP
}

\author{
A. Marciniak $^{1,2}$ \\ ${ }^{1}$ Institute of Computing Science \\ Poznań University of Technology \\ Piotrowo 2, 60-965 Poznań, Poland \\ E-mail: andrzej.marciniak@put.poznan.pl \\ ${ }^{2}$ Department of Computer Science \\ State University of Applied Sciences in Kalisz. \\ Poznańska 201-205, 62-800 Kalisz, Poland
}

Received: 14 May 2019; revised: 11 June 2019; accepted: 26 June 2019; published online: 30 June 2019

\begin{abstract}
In the paper we compare Nakao's method to our interval difference scheme of second order. Repeating some computational examples of Nakao, we have observed that our implementation of his method gives better results. Moreover, it appears that the presented interval difference scheme gives better enclosures of exact solutions than Nakao's method. Wę also point out that the considered interval method can be used to solve the Poisson equation with Dirichlet's condition, for which Nakao's method is not applicable.
\end{abstract}

Key words: Nakao's method, interval difference methods, elliptic boundary value problem, floating-point interval arithmetic

\section{INTRODUCTION}

Applying an approximate method to solve a problem on a computer we introduce three kinds of errors: representation errors and rounding errors (caused by floating-point arithmetic) and the error of method (usually called the truncation error). Verified numerical computing requires a mathematical tool to describe operations performed on computers. Such a mathematical tool, called interval arithmetic, has been developed by R.E. Moore in 1966 [1, 2] and extended by other researchers in the following years (see, e.g., [3-5]). Using interval methods (based on interval arithmetic) realized in interval floating-point arithmetic we can obtain solutions (in the form of intervals) which contain all mentioned errors.

The first approach to solve elliptic boundary value problems in interval arithmetic has been done by M.T. Nakao in 1988 [6] and extended in the following years [7, 8]. His method is based on Galerkin's approximation and finite elements methods known from conventional theory for solving elliptical problems (see, e.g., [9] and [10]). In [6] Nakao has presented a number of numerical examples for his method. We have repeated these experiments and obtained much better enclosures of the exact solution (see Sec. V. for details). Although Nakao's method can be applied to a lot of elliptic boundary value problems, there are some small inconveniences, from which the main one consists in nonapplicability of the method to the well-known Poisson equation.

In our previous paper we have considered interval difference methods of second and four order for solving the Poisson equation with Dirichlet's conditions in interval proper and direct floating-point arithmetic [11-15]. In this paper we generalize our second order method to solve the problems considered by Nakao in [6]. It appears that the presented interval difference scheme gives better enclosures of exact solutions than Nakao's method. However, it should be added that the proposed interval method can be considered only as an experimental one. Since some quantities occurring in the method are adopted experimentally (see Sec. IV. for details), 
a strictly mathematical proof that the obtained intervals contain exact solutions is rather impossible to receive.

The paper is divided into six sections. In Sec. II. we recall shortly the elliptic boundary value problem with the Dirichlet boundary conditions, for which Nakao's and our methods is applied. Since the main aim of this paper is to compare Nakao's and our methods, in Sec. III. we present in full details (i.e., in the form that can be implemented immediately) Nakao's method. In Sec. IV. we derive in details our interval difference scheme of second order. In Sec. V. we consider three numerical examples. The first two examples concern examples presented by Nakao in [6], and we compare his and our implementation with the results obtained by the interval method presented. The third example concerns an elliptical problem for which Nakao's method is not applicable. All these examples have been carried out in interval floatingpoint arithmetic using our IntervalArithmetic32and64 unit [16] written in the Delphi Pascal programming language. Finally, in Sec. VI. we shortly present some conclusions.

\section{THE ELLIPTIC BOUNDARY VALUE PROBLEM}

In this paper we restrict our considerations to the following elliptic boundary value problem of second order:

$$
\Delta u+c u=-f \text { in } \Omega, \quad u=g \text { on } \partial \Omega,
$$

where $\Omega=[0, \alpha] \times[0, \beta], u=u(x, y) \in C^{2}(\Omega)\left(C^{2}(\Omega)\right.$ means the space of all continuous real-valued functions defined on $\Omega$ such that $\Delta u$ is also continuous on $\Omega$ ), $c=$ $=c(x, y) \in L^{\infty}(\Omega), f=f(x, y) \in L^{2}(\Omega)$ (for definitions of $L^{\infty}(\Omega)$ and $L^{2}(\Omega)$ see the next section),

$$
\begin{array}{r}
\partial \Omega=\{(x, y): x=0, \alpha \text { and } 0 \leq y \leq \beta \\
\text { or } 0 \leq y \leq \alpha \text { and } y=0, \beta\},
\end{array}
$$

$\Delta$ is the Laplace operator defined by

$$
\begin{gathered}
\Delta u=\frac{\partial^{2} u}{\partial x^{2}}+\frac{\partial^{2} u}{\partial y^{2}} \\
g=g(x, y)= \begin{cases}g_{1}(y), & \text { for } x=0, \\
g_{2}(x), & \text { for } y=0 \\
g_{3}(y), & \text { for } x=\alpha, \\
g_{4}(x), & \text { for } y=\beta,\end{cases}
\end{gathered}
$$

and where

$$
\begin{aligned}
& g_{1}(0)=g_{2}(0), \quad g_{2}(\alpha)=g_{3}(0), \\
& g_{3}(\beta)=g_{4}(\alpha), \quad g_{4}(0)=g_{1}(\beta) .
\end{aligned}
$$

The conditions (2) are called the Dirichlet boundary conditions.

\section{NAKAO'S METHOD}

Nakao's method is an iteration method to find intervals containing exact solutions. In [6] Nakao has considered the problem (we resemble it in two-dimensional case)

$$
\begin{gathered}
\Delta u+b \cdot \nabla u+c u=-f \text { in } \Omega, \\
u=0 \text { on } \partial \Omega,
\end{gathered}
$$

where $\Delta$ denotes, as previously, the Laplace operator, $c$ and $f$ are the same functions as in (1),

$$
\nabla u=\left(\frac{\partial u}{\partial x}, \frac{\partial u}{\partial y}\right)^{T}
$$

and $b=\left(b_{1}, b_{2}\right)$. We assume that $b_{i}=b_{i}(x, y) \in W_{\infty}^{1}(\Omega)$ $(i=1,2)$, where $W_{\infty}^{1}(\Omega)$ denotes the usual $L^{\infty}-$ Sobolev space of first order on $\Omega$. The above problem can be rewritten in the so called weak form as follows:

$$
(\Delta u, \nabla \varphi)=(b \nabla u+c u, \varphi)+(f, \varphi), \quad \varphi \in H_{0}^{1}(\Omega) .
$$

Nakao has proved that the unique solution, i.e., the function $u=u(x, y)$, lies in the set $u_{h}+[\alpha]$, where for a given number $\alpha>0$ we have

$[\alpha]=\left\{\varphi \in H_{0}^{1}(\Omega):\|\varphi\|_{H_{0}^{1}(\Omega)} \leq \alpha,\|\varphi\|_{L^{2}(\Omega)} \leq C h \alpha\right\}$, and where $h$ is a parameter (mesh size) with $0<h<1$, while $C$ denotes a constant independent of $h$. In (3) the symbol $(\cdot, \cdot)$ means the inner product on $L^{2}(\Omega)$, where (in the case of two-dimensional problem)

$$
\begin{aligned}
L^{2}(\Omega)=\{u & \left.: \int_{\Omega}|u(x, y)|^{2} d x d y<\infty\right\}, \\
\|u\|_{L^{2}(\Omega)} & =\sqrt{\int_{\Omega}|u(x, y)|^{2} d x d y,} \\
(u, v) & =\int_{\Omega} u(x, y) v(x, y) d x d y,
\end{aligned}
$$

and $H_{0}^{1}(\Omega)$ is a kind of Sobolev space:

$$
H_{0}^{1}(\Omega)=\left\{u \in L^{2}(\Omega): \frac{\partial u}{\partial x}, \frac{\partial u}{\partial y} \in L^{2}(\Omega), u=0 \text { on } \partial \Omega\right\} .
$$

The quantities $u_{h}$ and $\alpha$ are calculated by iterations. Assuming $b=0^{1}$, we have

$$
\begin{aligned}
\left(\nabla u_{h}^{(k)}, \nabla \varphi_{i j}\right)= & \left(c u_{h}^{(k-1)}+f, \varphi_{i j}\right)+ \\
& +[-1,1] C h \alpha^{(k-1)}\left\|\varphi_{i j}\right\|_{L^{2}(\Omega)}, \\
\alpha^{(k)}= & C h\left(\left\|c u_{h}^{(k-1)}+f\right\|_{L^{2}(\Omega)}+\right. \\
& \left.+C h\|c\|_{L^{\infty}(\Omega)} \alpha^{(k-1)}\right),
\end{aligned}
$$

${ }^{1}$ This assumption is taking into account according to the examples presented by Nakao in [6] to whom we compare our interval difference scheme. 
where $L^{\infty}(\Omega)$ means the space consisting of functions $c=c(x, y)$ defined on $\Omega$ such that $|c(x, y)|$ has finite essential supremum on $\Omega$ (namely, there exists a positive constant $M$ such that $|c(x, y)| \leq M$ for almost every $(x, y)$ in $\Omega$, and the smallest such number $M$ is called the essential supremum of $|c(x, y)|$, and we write $M=$ $\left.=\operatorname{ess} \cdot \sup _{(x, y) \in \Omega}|c(x, y)|\right)$. The space $L^{\infty}(\Omega)$ is equipped with the norm

$$
\|c\|_{L^{\infty}(\Omega)}=\operatorname{ess} . \sup _{(x, y) \in \Omega}|c(x, y)| .
$$

Assuming that $\Omega=[0,1] \times[0,1]$ and partitioning the intervals $[0,1]$ in $x$ - and $y$-directions into $n$ equal parts of width $h$, the functions $\varphi_{i j}=\varphi_{i j}(x, y)$ denote the finite element basis functions associated with the interior nodes $\left(x_{i}, y_{j}\right)=(i h, j h)(i, j=1,2, \ldots, n-1)$ defined as follows:

$$
\varphi_{i j}(x, y)=\left\{\begin{array}{l}
1+i+j-\frac{x+y}{h}, \\
i h \leq x \leq(i+1) h, j h \leq y \leq(i+j+1) h-x, \\
1+j-\frac{y}{h}, \\
(i-1) h \leq x \leq i h,(i+j) h-x \leq y \leq(j+1) h, \\
1-i+\frac{x}{h} \\
(i-1) h \leq x \leq i h, j h \leq y \leq(i+j) h-x, \\
1-i-j+\frac{x+y}{h}, \\
(i-1) h \leq x \leq i h,(i+j-1) h-x \leq y \leq j h, \\
1-j+\frac{y}{h}, \\
i h \leq x \leq(i+1) h,(j-1) h \leq y \leq(i+j) h-x, \\
1+i-\frac{x}{h}, \\
i h \leq x \leq(i+1) h,(i+j) h-x \leq y \leq j h, \\
0, \quad \text { otherwise. }
\end{array}\right.
$$

In (4) we usually take $C=1$ (see [6, p. 327]). Since for the functions $\varphi_{i j}(x, y)$ given by (5) we have

$$
\left\|\varphi_{i j}\right\|_{L^{2}(\Omega)}=\frac{\sqrt{2}}{2} h
$$

the method (4) can be written in the form

$$
\begin{gathered}
\left(\nabla u_{h}^{(k)}, \nabla \varphi_{i j}\right)=\left(c u_{h}^{(k-1)}+f, \varphi_{i j}\right)+ \\
+[-1,1] \frac{\sqrt{2}}{2} h^{2} \alpha^{(k-1)}, \\
\alpha^{(k)}=h\left(\left\|c u_{h}^{(k-1)}+f\right\|_{L^{2}(\Omega)}+h\|c\|_{L^{\infty}(\Omega)} \alpha^{(k-1)}\right) .
\end{gathered}
$$

In the method (6) the quantities

$$
u_{h}^{(k)}=\sum_{i=1}^{n-1} \sum_{j=1}^{n-1} u_{i j}^{(k)} \varphi_{i j}(x, y)
$$

are intervals since the coefficients $u_{i j}^{(k)}=\left[\underline{A}_{i j}^{(k)}, \bar{A}_{i j}^{(k)}\right]$ are intervals, and the quantity $\alpha^{(k)}$ is a vector with real number components. Thus, the first formula in (6) is a system of linear equations of the form

$$
\left[\begin{array}{ccccccc}
\mathbf{A} & \mathbf{B} & \mathbf{0} & \cdots & \mathbf{0} & \mathbf{0} & \mathbf{0} \\
\mathbf{B} & \mathbf{A} & \mathbf{B} & \cdots & \mathbf{0} & \mathbf{0} & \mathbf{0} \\
\mathbf{0} & \mathbf{B} & \mathbf{A} & \ddots & \mathbf{0} & \mathbf{0} & \mathbf{0} \\
\vdots & \vdots & \ddots & \ddots & \ddots & \vdots & \vdots \\
\mathbf{0} & \mathbf{0} & \mathbf{0} & \ddots & \mathbf{A} & \mathbf{B} & \mathbf{0} \\
\mathbf{0} & \mathbf{0} & \mathbf{0} & \cdots & \mathbf{B} & \mathbf{A} & \mathbf{B} \\
\mathbf{0} & \mathbf{0} & \mathbf{0} & \cdots & \mathbf{0} & \mathbf{B} & \mathbf{A}
\end{array}\right]\left[\begin{array}{c}
\mathbf{u}_{1}^{(k)} \\
\mathbf{u}_{2}^{(k)} \\
\mathbf{u}_{3}^{(k)} \\
\vdots \\
\mathbf{u}_{n-3}^{(k)} \\
\mathbf{u}_{n-2}^{(k)} \\
\mathbf{u}_{n-1}^{(k)}
\end{array}\right]=\left[\begin{array}{c}
\mathbf{d}_{1}^{(k)} \\
\mathbf{d}_{2}^{(k)} \\
\mathbf{d}_{3}^{(k)} \\
\vdots \\
\mathbf{d}_{n-3}^{(k)} \\
\mathbf{d}_{n-2}^{(k)} \\
\mathbf{d}_{n-1}^{(k)}
\end{array}\right],
$$

$$
\begin{aligned}
\mathbf{A} & =\left[\begin{array}{lllllll}
a & b & 0 & \cdots & 0 & 0 & 0 \\
b & a & b & \cdots & 0 & 0 & 0 \\
0 & b & a & \ddots & 0 & 0 & 0 \\
\vdots & \vdots & \ddots & \ddots & \ddots & \vdots & \vdots \\
0 & 0 & 0 & \ddots & a & b & 0 \\
0 & 0 & 0 & \cdots & b & a & b \\
0 & 0 & 0 & \cdots & 0 & b & a
\end{array}\right], \\
\mathbf{B} & =\left[\begin{array}{lllll}
b & 0 & \cdots & 0 & 0 \\
0 & b & \cdots & 0 & 0 \\
\vdots & \vdots & \ddots & \vdots & \vdots \\
0 & 0 & \cdots & b & 0 \\
0 & 0 & \cdots & 0 & b
\end{array}\right],
\end{aligned}
$$

$$
\begin{gathered}
\mathbf{u}_{i}^{(k)}=\left[\begin{array}{c}
u_{i 1}^{(k)} \\
u_{i 2}^{(k)} \\
\vdots \\
u_{i, n-1}^{(k)}
\end{array}\right], \quad \mathbf{d}_{i}^{(k)}=\left[\begin{array}{c}
d_{i 1}^{(k)} \\
d_{i 2}^{(k)} \\
\vdots \\
d_{i, n-1}^{(k)}
\end{array}\right] \\
i=1,2, \ldots, n-1 .
\end{gathered}
$$

where

$$
\begin{gathered}
a=\frac{4}{h^{2}}, \quad b=-\frac{1}{h^{2}}, \\
d_{i j}^{(k)}=\frac{1}{h^{2}}\left(I_{i j 4}^{c} u_{i-1, j}^{(k-1)}+I_{i j 6}^{c} u_{i-1, j+1}^{(k-1)}+I_{i j 2}^{c} u_{i, j-1}^{(k-1)}+\right. \\
\left.+I_{i j 1}^{c} u_{i j}^{(k-1)}+I_{i j 3}^{c} u_{i, j+1}^{(k-1)}+I_{i j 7}^{c} u_{i+1, j-1}^{(k-1)}+I_{i j 5}^{c} u_{i+1, j}^{(k-1)}\right)+ \\
+\frac{1}{h^{2}} f_{i j}+[-1,1] \frac{\sqrt{2}}{2} \alpha_{i j}^{(k-1)}, \quad \begin{array}{l}
i, j=1,2, \ldots, n-1,
\end{array}
\end{gathered}
$$


and where $u_{0 j}^{(k-1)}=u_{n j}^{(k-1)}=u_{i 0}^{(k-1)}=u_{i n}^{(k-1)}=0$,

$$
\begin{aligned}
f_{i j}= & \int_{0}^{1} \int_{0}^{1} f(x, y) \varphi_{i j}(x, y) d x d y=\int_{(i-1) h}^{(i+1) h} \int_{j h}^{(i+j+1) h-x} f(x, y)\left[1+i+j-\frac{1}{h}(x+y)\right] d y d x+ \\
& +\int_{(i-1) h}^{i h} \int_{(i+j) h-x}^{(j+1) h} f(x, y)\left(i+j-\frac{y}{h}\right) d y d x+\int_{(i-1) h}^{i h}\left(1-i+\frac{x}{h}\right) \int_{j h}^{(i+j) h-x} f(x, y) d y d x+ \\
& +\int_{(i-1) h}^{i h} \int_{(i+1) h}^{j h} f(x, y)\left[1-i-j+\frac{1}{h}(x+y)\right] d y d x+\int_{i h}^{(i+1) h} \int_{(j-1) h}^{(i+j) h-x} f(x, y)\left(1-j+\frac{y}{h}\right) d y d x+ \\
& +\int_{i h}^{j h}\left(1+i-\frac{x}{h}\right) \int_{(i+j) h-x}^{j h} f(x, y) d y d x,
\end{aligned}
$$

For $I_{i j l}^{c}(l=1,2, \ldots, 7)$ we have

$$
\begin{aligned}
& I_{i j 1}^{c}=\int_{0}^{1} \int_{0}^{1} c(x, y) \varphi_{i j}^{2}(x, y) d x d y=\int_{i h}^{(i+1) h} \int_{j h}^{(i+j+1) h-x} c(x, y)\left[1+i+j-\frac{1}{h}(x+y)\right]^{2} d y d x+ \\
& +\int_{(i-1) h}^{i h} \int_{(i+j) h-x}^{(j+1) h} c(x, y)\left(1+j-\frac{y}{h}\right)^{2} d y d x+\int_{(i-1) h}^{i h}\left(1-i+\frac{x}{h}\right)^{2} \int_{j h}^{(i+j) h-x} c(x, y) d y d x+ \\
& +\int_{(i-1) h}^{i h} \int_{(i+j-1) h-x}^{j h} c(x, y)\left[1-i-j+\frac{1}{h}(x+y)\right]^{2} d y d x+\int_{i h}^{(i+1) h} \int_{(j-1) h}^{(i+j) h-x} c(x, y)\left(1-j+\frac{y}{h}\right)^{2} d y d x+ \\
& +\int_{i h}^{(i+1) h}\left(1+i-\frac{x}{h}\right)^{2} \int_{(i+j) h-x}^{j h} c(x, y) d y d x \\
& I_{i j 2}^{c}=\int_{0}^{1} \int_{0}^{1} c(x, y) \varphi_{i j}(x, y) \varphi_{i, j-1}(x, y) d x d y=\int_{(i-1) h}^{i h} \int_{(i+1-1) h-x}^{j h} c(x, y)\left[1-i-j+\frac{1}{h}(x+y)\right]\left(j-\frac{y}{h}\right) d y d x+ \\
& +\int_{i h}^{(i+1) h} \int_{(j-1) h}^{(i+j) h-x} c(x, y)\left(1-j+\frac{y}{h}\right)\left[i+j-\frac{1}{h}(x+y)\right] d y d x \\
& I_{i j 3}^{c}=\int_{0}^{1} \int_{0}^{1} c(x, y) \varphi_{i j}(x, y) \varphi_{i, j+1}(x, y) d x d y=\int_{i h}^{(i+1) h} \int_{\substack{j h \\
(j+1) h}}^{(i+j+1) h-x} c(x, y)\left[1+i+j-\frac{1}{h}(x+y)\right]\left(-j+\frac{y}{h}\right) d y d x+ \\
& +\int_{(i-1) h}^{i h} \int_{(i+j) h-x}^{(j+1) h} c(x, y)\left(1+j-\frac{y}{h}\right)\left[-i-j+\frac{1}{h}(x+y)\right] d y d x \\
& I_{i j 4}^{c}=\int_{0}^{1} \int_{0}^{1} c(x, y) \varphi_{i j}(x, y) \varphi_{i-1, j}(x, y) d x d y=\int_{(i-1) h}^{i h}\left(1-i+\frac{x}{h}\right) \int_{j h}^{(i+j) h-x} c(x, y)\left[i+j-\frac{1}{h}(x+y)\right] d y d x+ \\
& +\int_{(i-1) h}^{i h}\left(i-\frac{x}{h}\right) \int_{(i+j-1) h-x}^{j h} c(x, y)\left[1-i-j+\frac{1}{h}(x+y)\right] d y d x,
\end{aligned}
$$




$$
\begin{aligned}
& I_{i j 5}^{c}=\int_{0}^{1} \int_{0}^{1} c(x, y) \varphi_{i j}(x, y) \varphi_{i+1, j}(x, y) d x d y=\int_{i h}^{(i+1) h}\left(-i+\frac{x}{h}\right)^{(i+j+1) h-x} \int_{j h}^{c} c(x, y)\left[1+i+j-\frac{1}{h}(x+y)\right] d y d x+ \\
& +\int_{i h}^{(i+1) h}\left(1+i-\frac{x}{h}\right) \int_{(i+j) h-x}^{j h} c(x, y)\left[-i-j+\frac{1}{h}(x+y)\right] d y d x \\
& I_{i j 6}^{c}=\int_{0}^{1} \int_{0}^{1} c(x, y) \varphi_{i j}(x, y) \varphi_{i-1, j+1}(x, y) d x d y=\int_{(i-1) h}^{i h}\left(i-\frac{x}{h}\right) \int_{(i+j) h-x}^{(j+1) h} c(x, y)\left(1+j-\frac{y}{h}\right) d y d x+ \\
& +\int_{(i-1) h}^{i h}\left(1-i+\frac{x}{h}\right) \int_{j h}^{(i+j) h-x} c(x, y)\left(-j+\frac{y}{h}\right) d y d x \\
& I_{i j 7}^{c}=\int_{0}^{1} \int_{0}^{1} c(x, y) \varphi_{i j}(x, y) \varphi_{i+1, j-1}(x, y) d x d y=\int_{i h}^{(i+1) h}\left(-i+\frac{x}{h}\right) \int_{(j-1) h}^{(i+j) h-x} c(x, y)\left(1-j+\frac{y}{h}\right) d y d x+ \\
& +\int_{i h}^{(i+1) h}\left(1+i-\frac{x}{h}\right) \int_{(i+j) h-x}^{j h} c(x, y)\left(j-\frac{y}{h}\right) d y d x .
\end{aligned}
$$

The formula for $\alpha^{(k)}$ contains two norms. The first one is as follows:

$$
\left\|c u_{h}^{(k-1)}+f\right\|_{L^{2}(\Omega)}=\sqrt{\int_{0}^{1} \int_{0}^{1}\left(c u_{h}^{(k-1)}+f(x, y)\right)^{2} d x d y .}
$$

Since $\alpha^{(k)}$ is a vector, this norm should be calculated for each $i$ and $j$. Taking into account (5) and (7), and denoting the expression under the square root in (16) by $\beta_{i j}$, we have

$$
\beta_{i j}=\beta_{i j}^{(1)}+2 \beta_{i j}^{(2)}+\int_{0}^{1} \int_{0}^{1} f^{2}(x, y) d x d y
$$

where

$$
\begin{aligned}
\beta_{i j}^{(1)}= & \left(u_{i j}^{(k-1)}\right)^{2}\left\{\int_{i h}^{(i+1) h} \int_{j h}^{(i+j+1) h-x} c^{2}(x, y)\left[1+i+j-\frac{1}{h}(x+y)\right]^{2} d y d x+\right. \\
& +\int_{(i-1) h} \int_{(i+j) h-x}^{i h} c^{2}(x, y)\left(1+j-\frac{y}{h}\right)^{2} d y d x+\int_{(i-1) h}^{(j+1) h}\left(1-i+\frac{x}{h}\right)^{2} \int_{j h}^{(i+j) h-x} c^{2}(x, y) d y d x+ \\
& +\int_{(i-1) h}^{i h} \int_{(i+j) h}^{j h} c^{2}(x, y)\left[1-i-j+\frac{1}{h}(x+y)\right]^{2} d y d x+\int_{i h}^{(i+1) h} \int_{(j-1) h}^{(i+j) h-x} c^{2}(x, y)\left(1-j+\frac{y}{h}\right)^{2} d y d x+ \\
& \left.+\int_{i h}^{\left(1+i-\frac{x}{h}\right)^{2}} \int_{(i+j) h-x}^{j} c^{2}(x, y) d y d x\right\}
\end{aligned}
$$




$$
\begin{aligned}
\beta_{i j}^{(2)}= & u_{i j}^{(k-1)}\left\{\int_{i h}^{(i+1) h} \int_{j h}^{(i+j+1) h-x} c(x, y)\left[1+i+j-\frac{1}{h}(x+y)\right] f(x, y) d y d x+\right. \\
& +\int_{(i-1) h}^{i h} \int_{(i+j) h-x}^{(j+1) h} c(x, y)\left(1+j-\frac{y}{h}\right) f(x, y) d y d x+\int_{(i-1) h}^{i h}\left(1-i+\frac{x}{h} \int_{j h}^{(i+j) h-x} c(x, y) f(x, y) d y d x+\right. \\
& +\int_{(i-1) h}^{i h} \int_{(i+j-1) h-x}^{j h} c(x, y)\left[1-i-j+\frac{1}{h}(x+y)\right] f(x, y) d y d x+\int_{i h}^{(i+1) h} \int_{(j-1) h}^{(i+j) h-x} c(x, y)\left(1-j+\frac{y}{h}\right) f(x, y) d y d x+ \\
& \left.+\int_{i h}^{(1+1) h}\left(1+i-\frac{x}{h}\right) \int_{(i+j) h-x}^{j h} c(x, y) f(x, y) d y d x\right\} .
\end{aligned}
$$

The second norm, i.e.,

$$
\begin{aligned}
\|c\|_{L^{\infty}(\Omega)} & =\|c(x, y)\|_{L^{\infty}(\Omega)}= \\
& =\operatorname{ess.sup}_{(x, y) \in(0,1) \times(0,1)}|c(x, y)|,
\end{aligned}
$$

depends on $c(x, y)$ and should be calculated for each problem separately. Thus, we have

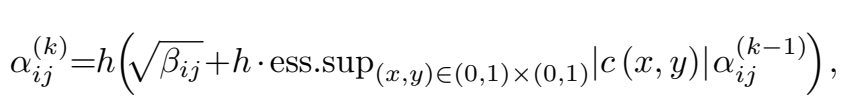

where $\beta_{i j}$ is given by (16).

In order to find integrals (8)-(15) and also those occurring in (17) we usually apply quadratures. But such an ap- proach causes additional errors (errors of quadratures) in obtained results. Thus, whenever it is possible, we recommend to find analytical forms of these integrals (see examples in Sec. V.). A mathematical software, e.g., Mathematica, Matlab or Derive, can be helpful in this case.

For initial values in process (6) we take the solution $u_{h}^{(0)}$ obtained from the Galerkin approximation, i.e.,

$\left(\nabla u_{h}^{(0)}, \nabla \varphi_{i j}\right)=\left(c u_{h}^{(0)}+f, \varphi_{i j}\right), i, j=1,2, \ldots, n-1$, and $\alpha^{(0)}=\left(\alpha_{11}^{(0)}, \alpha_{12}^{(0)}, \ldots, \alpha_{n-1, n-1}^{(0)}\right)^{T}=(0,0, \ldots, 0)^{T}$. The approximation (18) consists in finding the solution of the following system of linear equations:

$$
\left[\begin{array}{ccccccc}
\mathbf{A}_{1} & \mathbf{B}_{1} & \mathbf{0} & \cdots & \mathbf{0} & \mathbf{0} & \mathbf{0} \\
\widehat{\mathbf{B}}_{2} & \mathbf{A}_{2} & \mathbf{B}_{2} & \cdots & \mathbf{0} & \mathbf{0} & \mathbf{0} \\
\mathbf{0} & \widehat{\mathbf{B}}_{3} & \mathbf{A}_{3} & \ddots & \mathbf{0} & \mathbf{0} & \mathbf{0} \\
\vdots & \vdots & \ddots & \ddots & \ddots & \vdots & \vdots \\
\mathbf{0} & \mathbf{0} & \mathbf{0} & \ddots & \mathbf{A}_{n-3} & \mathbf{B}_{n-3} & v \\
\mathbf{0} & \mathbf{0} & \mathbf{0} & \cdots & \widehat{\mathbf{B}}_{n-2} & \mathbf{A}_{n-2} & \mathbf{B}_{n-2} \\
\mathbf{0} & \mathbf{0} & \mathbf{0} & \cdots & \mathbf{0} & \widehat{\mathbf{B}}_{n-1} & \mathbf{A}_{n-1}
\end{array}\right]\left[\begin{array}{c}
\mathbf{u}_{1}^{(0)} \\
\mathbf{u}_{2}^{(0)} \\
\mathbf{u}_{3}^{(0)} \\
\vdots \\
\mathbf{u}_{n-3}^{(0)} \\
\mathbf{u}_{n-2}^{(0)} \\
\mathbf{u}_{n-1}^{(0)}
\end{array}\right]=\left[\begin{array}{c}
\mathbf{d}_{1} \\
\mathbf{d}_{2} \\
\mathbf{d}_{3} \\
\vdots \\
\mathbf{d}_{n-3} \\
\mathbf{d}_{n-2} \\
\mathbf{d}_{n-1}
\end{array}\right]
$$

where

$$
\mathbf{A}_{i}=\left[\begin{array}{ccccccc}
a_{i 1} & a_{i 2}^{+} & 0 & \cdots & 0 & 0 & 0 \\
a_{i 1}^{-} & a_{i 2} & a_{i 3}^{+} & \cdots & 0 & 0 & 0 \\
0 & a_{i 2}^{-} & a_{i 3} & \ddots & 0 & 0 & 0 \\
\vdots & \vdots & \ddots & \ddots & \ddots & \vdots & \vdots \\
0 & 0 & 0 & \ddots & a_{i, n-3} & a_{i, n-2}^{+} & 0 \\
0 & 0 & 0 & \cdots & a_{i, n-3}^{-} & a_{i, n-2} & a_{i, n-1}^{+} \\
0 & 0 & 0 & \cdots & 0 & a_{i, n-2}^{-} & a_{i, n-1}
\end{array}\right]
$$




$$
\begin{gathered}
\mathbf{B}_{i}=\left[\begin{array}{ccccccc}
b_{i+1,1} & 0 & 0 & \cdots & 0 & 0 & 0 \\
b_{i+1,1}^{-} & b_{i+1,2} & 0 & \cdots & 0 & 0 & 0 \\
0 & b_{i+1,2}^{-} & b_{i+1,3} & \ddots & 0 & 0 & 0 \\
\vdots & \vdots & \ddots & \ddots & \ddots & \vdots & \vdots \\
0 & 0 & 0 & \ddots & b_{i+1, n-3} & 0 & 0 \\
0 & 0 & 0 & \cdots & b_{i+1, n-3}^{-} & b_{i+1, n-2} & 0 \\
0 & 0 & 0 & \cdots & 0 & b_{i+1, n-2}^{-} & b_{i+1, n-1}
\end{array}\right], \\
\widehat{\mathbf{B}}_{i}=\left[\begin{array}{ccccccc}
\widehat{b}_{i-1,1} & \widehat{b}_{i-1,2}^{+} & 0 & \cdots & 0 & 0 & 0 \\
0 & \widehat{b}_{i-1,2} & \widehat{b}_{i-1,3}^{+} & \cdots & 0 & 0 & 0 \\
0 & 0 & \widehat{b}_{i-1,3} & \ddots & 0 & 0 & 0 \\
\vdots & \vdots & \ddots & \ddots & \ddots & \vdots & \vdots \\
0 & 0 & 0 & \ddots & \widehat{b}_{i-1, n-3} & \widehat{b}_{i-1, n-2}^{+} & 0 \\
0 & 0 & 0 & \cdots & 0 & \widehat{b}_{i-1, n-2} & \widehat{b}_{i-1, n-1}^{+} \\
0 & 0 & 0 & \cdots & 0 & 0 & \widehat{b}_{i-1, n-1}
\end{array}\right],
\end{gathered}
$$

$$
\begin{aligned}
& \mathbf{u}_{i}^{(0)}=\left[\begin{array}{c}
u_{i 1}^{(0)} \\
u_{i 2}^{(0)} \\
\vdots \\
u_{i, n-1}^{(0)}
\end{array}\right], \quad \mathbf{d}_{i}=\left[\begin{array}{c}
d_{i 1} \\
d_{i 2} \\
\vdots \\
d_{i, n-1}
\end{array}\right] \\
& i=1,2, \ldots, n-1, \\
& a_{i j}=\frac{1}{h^{2}}\left(4-I_{i j 1}^{c}\right), \quad i, j=1,2, \ldots, n-1, \\
& a_{i j}^{-}=-\frac{1}{h^{2}}\left(1+I_{i j 2}^{c}\right), i=1,2, \ldots, n-1, j=1,2, \ldots, n-2, \\
& a_{i j}^{+}=-\frac{1}{h^{2}}\left(1+I_{i j 3}^{c}\right), i=1,2, \ldots, n-1, j=2,3, \ldots, n-1, \\
& b_{i+1, j}=-\frac{1}{h^{2}}\left(1+I_{i j 5}^{c}\right), i=1,2, \ldots, n-2, j=1,2, \ldots, n-1, \\
& b_{i+1, j}^{-}=-\frac{1}{h^{2}} I_{i j 7}^{c}, i=1,2, \ldots, n-2, j=1,2, \ldots, n-2, \\
& \widehat{b}_{i-1, j}=-\frac{1}{h^{2}}\left(1+I_{i j 4}^{c}\right), i=2,3, \ldots, n-1, j=1,2, \ldots, n-1, \\
& \widehat{b}_{i-1, j}^{+}=-\frac{1}{h^{2}} I_{i j 6}^{c}, i=2,3, \ldots, n-1, j=2,3, \ldots, n-1, \\
& d_{i j}=\frac{1}{h^{2}} f_{i j}, \quad i, j=1,2, \ldots, n-1,
\end{aligned}
$$

and where $I_{i j l}^{c}(l=1,2, \ldots, 7)$ are given by (9)-(15), and $f_{i j}$ - by (8).

The iterations (4) are stopped if for sufficiently small $\varepsilon>0$ and a positive integer $N$ we attain estimates:

$$
\begin{aligned}
\left\|u_{h}^{(N)}-u_{h}^{(N-1)}\right\|=\max _{i, j=1,2, \ldots, n-1}\left\{\left|\underline{A}_{i j}^{(N)}-\underline{A}_{i j}^{(N-1)}\right|,\right. & \\
& \left.\left|\bar{A}_{i j}^{(N)}-\bar{A}_{i j}^{(N-1)}\right|\right\}<\varepsilon
\end{aligned}
$$

and

$$
\max _{i, j=1,2, \ldots, n-1}\left|\alpha_{i j}^{(N)}-\alpha_{i j}^{(N-1)}\right|<\varepsilon .
$$

Finally, for an appropriate $\delta>0$, we set

$$
\widetilde{u}_{h}^{(N)}=\sum_{i=1}^{n-1} \sum_{j=1}^{n-1} \widetilde{A}_{i j}^{(N)} \varphi_{i j}(x, y), \quad \widetilde{\alpha}^{(N)}=\alpha^{(N)}+\delta,
$$

where $\widetilde{A}_{i j}^{(N)}=\left[\underline{A}_{i j}^{(N)}-\delta, \bar{A}_{i j}^{(N)}+\delta\right]$. This procedure is called $\delta$-extension and the intervals $\widetilde{A}_{i j}^{(N)}$ are enclosures for $u\left(x_{i}, y_{j}\right)$.

Although Nakao's method can be applied to a lot of elliptic boundary value problems, there are three small inconveniences:

- the mesh size $h$ must be the same in $x$-and $y$-directions (it would be more convenient to take $h$ for $x$-direction and $k \neq h$ for $y$-direction),

- the function $u=u(x, y)$ should be equal to 0 on the boundary $\partial \Omega$, while, in general, it can be $u(x, y)=$ $=g(x, y) \neq 0$ on this boundary,

- the method cannot be applied for $(b, c)=(0,0)$, which means that, e.g., the conventional Poisson equation cannot be solved. 
Let us note that for the method (6), where we have assumed $b=0$, if we take also $c=0$, then we have

$$
\begin{gathered}
\left(\nabla u_{h}^{(k)}, \nabla \varphi_{i j}\right)=\left(f, \varphi_{i j}\right)+[-1,1] \frac{\sqrt{2}}{2} h^{2} \alpha^{(k-1)}, \\
\alpha^{(k)}=h\|f\|_{L^{2}(\Omega)}=\mathrm{const}
\end{gathered}
$$

and no iteration has been carried out. The interval difference method developed by us are free from these inconveniences. Moreover, numerical experiments show that our method gives better enclosures (intervals with smaller widths) of the exact solutions.

\section{AN INTERVAL DIFFERENCE SCHEME OF SECOND ORDER}

For the problem (1) with $c=0$, interval difference methods of second order based on proper and directed interval arithmetic we have developed in details in [11-15]. ${ }^{2}$ In this section we expand our second order method in proper interval arithmetic for the case $c=c(x, y)$.

Partitioning the interval $[0, \alpha]$ into $n$ equal parts of width $h$ and the interval $[0, \beta]$ into $m$ equal parts of width $k$ provides a mean of placing a grid on the rectangle $[0, \alpha] \times[0, \beta]$ with mesh points $\left(x_{i}, y_{j}\right)$, where $h=\alpha / n, k=\beta / m .{ }^{3}$ Assuming that the fourth order partial derivatives of $u$ exist, for each mesh point in the interior of grid we use the Taylor series in the variable $x$ about $x_{i}$ and in the variable $y$ about $y_{j}$. This allows us to express the equation (1) at the points $\left(x_{i}, y_{j}\right)$ as

$\delta_{x}^{2} u_{i j}-\frac{h^{2}}{12} \frac{\partial^{4} u}{\partial x^{4}}\left(\xi_{i}, y_{j}\right)+\delta_{y}^{2} u_{i j}-\frac{k^{2}}{12} \frac{\partial^{4} u}{\partial y^{4}}\left(x_{i}, \eta_{j}\right)+c_{i j} u_{i j}=-f_{i j}$,

where

$$
\begin{aligned}
& \delta_{x}^{2} u_{i j}=\frac{u_{i+1, j}-2 u_{i, j}+u_{i-1, j}}{h^{2}}, \\
& \delta_{y}^{2} u_{i j}=\frac{u_{i, j+1}-2 u_{i, j}+u_{i, j-1}}{k^{2}},
\end{aligned}
$$

$i=1,2, \ldots, n-1 ; j=1,2, \ldots, m-1, u_{i j}=$ $=u\left(x_{i}, y_{j}\right), c_{i j}=c\left(x_{i}, y_{j}\right), f_{i j}=f\left(x_{i}, y_{j}\right)$, and where $\xi_{i} \in\left(x_{i-1}, x_{i+1}\right), \eta_{j} \in\left(y_{j-1}, y_{j+1}\right)$ are intermediate points, and the boundary conditions as

$$
\begin{array}{ll}
u\left(0, y_{j}\right)=g_{1}\left(y_{j}\right), & \text { for } j=0,1, \ldots, m, \\
u\left(x_{i}, 0\right)=g_{2}\left(x_{i}\right), & \text { for } i=1,2, \ldots, n-1, \\
u\left(\alpha, y_{j}\right)=g_{3}\left(y_{j}\right), & \text { for } j=0,1, \ldots, m, \\
u\left(x_{i}, \beta\right)=g_{4}\left(x_{i}\right), & \text { for } i=1,2, \ldots, n-1,
\end{array}
$$

Note that in our previous papers [11-15] on the right-hand side of (19) we wrote $f_{i j}$ instead of $-f_{i j}$. The modification in this paper is a consequence of Nakao's notation of elliptic boundary value problem used in [6].

Directly from (1) we have

$$
\begin{aligned}
& \frac{\partial^{4} u}{\partial x^{4}}=-\frac{\partial^{2} f}{\partial x^{2}}-\frac{\partial^{4} u}{\partial x^{2} \partial y^{2}}-\frac{\partial^{2} c}{\partial x^{2}} u-2 \frac{\partial c}{\partial x} \frac{\partial u}{\partial x}-c \frac{\partial^{2} u}{\partial x^{2}}, \\
& \frac{\partial^{4} u}{\partial y^{4}}=-\frac{\partial^{2} f}{\partial y^{2}}-\frac{\partial^{4} u}{\partial y^{2} \partial x^{2}}-\frac{\partial^{2} c}{\partial y^{2}} u-2 \frac{\partial c}{\partial y} \frac{\partial u}{\partial y}-c \frac{\partial^{2} u}{\partial y^{2}} .
\end{aligned}
$$

Assuming that $\frac{\partial^{4} u}{\partial x^{2} \partial y^{2}}=\frac{\partial^{4} u}{\partial y^{2} \partial x^{2}}$, and substituting (21) into (19) we get

$\delta_{x}^{2} u_{i j}+\delta_{y}^{2} u_{i j}+$

$+\frac{h^{2}}{12}\left[\frac{\partial^{2} f}{\partial x^{2}}\left(\xi_{i}, y_{j}\right)+\frac{\partial^{4} u}{\partial x^{2} \partial y^{2}}\left(\xi_{i}, y_{j}\right)+c\left(\xi_{i}, y_{j}\right) \frac{\partial^{2} u}{\partial x^{2}}\left(\xi_{i}, y_{j}\right)+\right.$

$\left.+\frac{\partial^{2} c}{\partial x^{2}}\left(\xi_{i}, y_{j}\right) u\left(\xi_{i}, y_{j}\right)+2 \frac{\partial c}{\partial x}\left(\xi_{i}, y_{j}\right) \frac{\partial u}{\partial x}\left(\xi_{i}, y_{j}\right)\right]+$

$+\frac{k^{2}}{12}\left[\frac{\partial^{2} f}{\partial y^{2}}\left(x_{i}, \eta_{j}\right)+\frac{\partial^{4} u}{\partial x^{2} \partial y^{2}}\left(x_{i}, \eta_{j}\right)+c\left(x_{i}, \eta_{j}\right) \frac{\partial^{2} u}{\partial y^{2}}\left(x_{i}, \eta_{j}\right)+\right.$

$\left.+\frac{\partial^{2} c}{\partial y^{2}}\left(x_{i}, \eta_{j}\right) u\left(x_{i}, \eta_{j}\right)+2 \frac{\partial c}{\partial y}\left(x_{i}, \eta_{j}\right) \frac{\partial u}{\partial y}\left(x_{i}, \eta_{j}\right)\right]+$

$+c_{i j} u_{i j}=-f_{i j}$.

But if the appropriate functions are bounded, then we can write

$$
\begin{aligned}
& c\left(\xi_{i}, y_{j}\right)=c_{i j}+O(h), \quad \frac{\partial^{2} c}{\partial x^{2}}\left(\xi_{i}, y_{j}\right)=\frac{\partial^{2} c_{i j}}{\partial x^{2}}+O(h), \\
& \frac{\partial^{2} u}{\partial x^{2}}\left(\xi_{i}, y_{j}\right)=\frac{\partial^{2} u}{\partial x^{2}}\left(x_{i}, y_{j}\right)+O(h)=\delta_{x}^{2} u_{i j}+O(h), \\
& u\left(\xi_{i}, y_{j}\right)=u_{i j}+O(h), \quad \frac{\partial c}{\partial x}\left(\xi_{i}, y_{j}\right)=\frac{\partial c_{i j}}{\partial x}+O(h), \\
& \frac{\partial u}{\partial x}\left(\xi_{i}, y_{j}\right)=\frac{\partial u}{\partial x}\left(x_{i}, y_{j}\right)+O(h)=\delta_{x} u_{i j}+O(h)
\end{aligned}
$$

and

$$
\begin{aligned}
& c\left(x_{i}, \eta_{j}\right)=c_{i j}+O(k), \quad \frac{\partial^{2} c}{\partial y^{2}}\left(x_{i}, \eta_{j}\right)=\frac{\partial^{2} c_{i j}}{\partial y^{2}}+O(k), \\
& \frac{\partial^{2} u}{\partial y^{2}}\left(x_{i}, \eta_{j}\right)=\frac{\partial^{2} u}{\partial y^{2}}\left(x_{i}, y_{j}\right)+O(k)=\delta_{y}^{2} u_{i j}+O(k), \\
& u\left(x_{i}, \eta_{j}\right)=u_{i j}+O(k), \quad \frac{\partial c}{\partial y}\left(x_{i}, \eta_{j}\right)=\frac{\partial c_{i j}}{\partial y}+O(k), \\
& \frac{\partial u}{\partial y}\left(x_{i}, \eta_{j}\right)=\frac{\partial u}{\partial y}\left(x_{i}, y_{j}\right)+O(k)=\delta_{y} u_{i j}+O(k),
\end{aligned}
$$

where

$$
\begin{aligned}
\frac{\partial^{2} c_{i j}}{\partial x^{2}} & =\frac{\partial^{2} c}{\partial x^{2}}\left(x_{i}, y_{j}\right), & & \frac{\partial^{2} c_{i j}}{\partial y^{2}}=\frac{\partial^{2} c}{\partial y^{2}}\left(x_{i}, y_{j}\right), \\
\frac{\partial c_{i j}}{\partial x} & =\frac{\partial c}{\partial x}\left(x_{i}, y_{j}\right), & \frac{\partial c_{i j}}{\partial y} & =\frac{\partial c}{\partial y}\left(x_{i}, y_{j}\right)
\end{aligned}
$$

\footnotetext{
${ }^{2}$ In [14] we have also presented interval difference methods of fourth order.

${ }^{3}$ When $f(x, y)=f(y, x)$, we usually take $h=k$. In this case $m=$ round $(n \beta / \alpha)$.
} 
and

$$
\delta_{x} u_{i j}=\frac{u_{i+1, j}-u_{i-1, j}}{2 h}, \quad \delta_{y} u_{i j}=\frac{u_{i, j+1}-u_{i, j-1}}{2 k} .
$$

Thus, the equation (22) can be written in the form

$$
\begin{aligned}
& \left(1+\frac{h^{2}}{12} c_{i j}\right) \delta_{x}^{2} u_{i j}+\left(1+\frac{k^{2}}{12} c_{i j}\right) \delta_{y}^{2} u_{i j}+\frac{h^{2}}{6} \frac{\partial c_{i j}}{\partial x} \delta_{x} u_{i j}+ \\
& +\frac{k^{2}}{6} \frac{\partial c_{i j}}{\partial y} \delta_{y} u_{i j}+\left(c_{i j}+\frac{h^{2}}{12} \frac{\partial^{2} c_{i j}}{\partial x^{2}}+\frac{k^{2}}{12} \frac{\partial^{2} c_{i j}}{\partial y^{2}}\right) u_{i j}+ \\
& +\frac{h^{2}}{12}\left[\frac{\partial^{2} f}{\partial x^{2}}\left(\xi_{i}, y_{j}\right)+\frac{\partial^{4} u}{\partial x^{2} \partial y^{2}}\left(\xi_{i}, y_{j}\right)\right]+ \\
& +\frac{k^{2}}{12}\left[\frac{\partial^{2} f}{\partial y^{2}}\left(x_{i}, \eta_{j}\right)+\frac{\partial^{4} u}{\partial x^{2} \partial y^{2}}\left(x_{i}, \eta_{j}\right)\right]+ \\
& +O\left(h^{3}\right)+O\left(k^{3}\right)=-f_{i j} .
\end{aligned}
$$

If $h=k$ and we take into account that

$$
\frac{\partial^{2} u}{\partial x^{2}}\left(x_{i}, y_{j}\right)+\frac{\partial^{2} u}{\partial y^{2}}\left(x_{i}, y_{j}\right)=-f_{i j}-c_{i j} u_{i j},
$$

which directly follows from (1), then we can write the equation (22) as

$$
\begin{aligned}
& \delta_{x}^{2} u_{i j}+\delta_{y}^{2} u_{i j}+\frac{h^{2}}{6}\left(\frac{\partial c_{i j}}{\partial x} \delta_{x} u_{i j}+\frac{\partial c_{i j}}{\partial y} \delta_{y} u_{i j}\right)+ \\
& +\left[c_{i j}\left(1-\frac{h^{2}}{12}\right)+\frac{h^{2}}{12}\left(\frac{\partial^{2} c_{i j}}{\partial x^{2}}+\frac{\partial^{2} c_{i j}}{\partial y^{2}}\right)\right] u_{i j}+ \\
& +\frac{h^{2}}{12}\left[-c_{i j} f_{i j}+\frac{\partial^{2} f}{\partial x^{2}}\left(\xi_{i}, y_{j}\right)+\frac{\partial^{2} f}{\partial y^{2}}\left(x_{i}, \eta_{j}\right)+\right. \\
& \left.+\frac{\partial^{4} u}{\partial x^{2} \partial y^{2}}\left(\xi_{i}, y_{j}\right)+\frac{\partial^{4} u}{\partial x^{2} \partial y^{2}}\left(x_{i}, \eta_{j}\right)\right]+O\left(h^{3}\right)=-f_{i j} .
\end{aligned}
$$

From (23) and (24) we have, respectively

$$
\begin{aligned}
& \left(1-\frac{h^{2}}{12} c_{i j}\right) \delta_{x}^{2} u_{i j}+\left(1-\frac{k^{2}}{12} c_{i j}\right) \delta_{y}^{2} u_{i j}+\frac{h^{2}}{6} \frac{\partial c_{i j}}{\partial x} \delta_{x} u_{i j}+ \\
& +\frac{k^{2}}{6} \frac{\partial c_{i j}}{\partial y} \delta_{y} u_{i j}+\left(c_{i j}+\frac{h^{2}}{12} \frac{\partial^{2} c_{i j}}{\partial x^{2}}+\frac{k^{2}}{12} \frac{\partial^{2} c_{i j}}{\partial y^{2}}\right) u_{i j}= \\
& =-f_{i j}-\frac{h^{2}}{12}\left[\frac{\partial^{2} f}{\partial x^{2}}\left(\xi_{i}, y_{j}\right)+\frac{\partial^{4} u}{\partial x^{2} \partial y^{2}}\left(\xi_{i}, y_{j}\right)\right]+ \\
& -\frac{k^{2}}{12}\left[\frac{\partial^{2} f}{\partial y^{2}}\left(x_{i}, \eta_{j}\right)+\frac{\partial^{4} u}{\partial x^{2} \partial y^{2}}\left(x_{i}, \eta_{j}\right)\right]+O\left(h^{3}\right)+O\left(k^{3}\right)
\end{aligned}
$$

and

$$
\begin{aligned}
& \delta_{x}^{2} u_{i j}+\delta_{y}^{2} u_{i j}+\frac{h^{2}}{6}\left(\frac{\partial c_{i j}}{\partial x} \delta_{x} u_{i j}+\frac{\partial c_{i j}}{\partial y} \delta_{y} u_{i j}\right)+ \\
& +\left[c_{i j}\left(1-\frac{h^{2}}{12} c_{i j}\right)+\frac{h^{2}}{12}\left(\frac{\partial^{2} c_{i j}}{\partial x^{2}}+\frac{\partial^{2} c_{i j}}{\partial y^{2}}\right)\right] u_{i j}= \\
& =\left(\frac{h^{2}}{12} c_{i j}-1\right) f_{i j}-\frac{h^{2}}{12}\left[\frac{\partial^{2} f}{\partial x^{2}}\left(\xi_{i}, y_{j}\right)+\frac{\partial^{2} f}{\partial y^{2}}\left(x_{i}, \eta_{j}\right)+\right. \\
& \left.\quad+\frac{\partial^{4} u}{\partial x^{2} \partial y^{2}}\left(\xi_{i}, y_{j}\right)+\frac{\partial^{4} u}{\partial x^{2} \partial y^{2}}\left(x_{i}, \eta_{j}\right)\right]+O\left(h^{3}\right) .
\end{aligned}
$$

To construct interval methods, let us assume that there exists a constant $M$ such that

$$
\left|\frac{\partial^{4} u}{\partial x^{2} \partial y^{2}}(x, y)\right| \leq M
$$

for all $0 \leq x \leq \alpha$ and $0 \leq y \leq \beta$. It is obvious that $\frac{\partial^{2} f}{\partial x^{2}}(\xi, y)+\frac{\partial^{4} u}{\partial x^{2} \partial y^{2}}(\xi, y) \in \Psi(X+[-h, h], Y)+[-M, M]$, $\frac{\partial^{2} f}{\partial y^{2}}(x, \eta)+\frac{\partial^{4} u}{\partial x^{2} \partial y^{2}}(x, \eta) \in \Omega(X, Y+[-k, k])+[-M, M]$, for any $\xi \in(x-h, x+h)$ and any $\eta \in(y-k, y+k)$, where $X$ and $Y$ denote interval extensions of $x$ and $y$, respectively, and $\Psi(X, Y)$ and $\Omega(X, Y)$ are interval extensions of $\frac{\partial^{2} f}{\partial x^{2}}(x, y)$ and $\frac{\partial^{2} f}{\partial y^{2}}(x, y)$, respectively. If we denote by $U(X, Y), F(X, Y), C(X, Y), D_{x} C(X, Y)$, $D_{y} C(X, Y), D_{x}^{2} C(X, Y)$ and $D_{y}^{2} C(X, Y)$ interval extensions of $u(x, y), f(x, y), c(x, y), \frac{\partial c}{\partial x}(x, y), \frac{\partial c}{\partial y}(x, y)$, $\frac{\partial^{2} c}{\partial x^{2}}(x, y)$ and $\frac{\partial^{2} c}{\partial y^{2}}(x, y)$, respectively, then it is easy to write interval analogies to equations (25) and (26). For (25) we have

$$
\begin{aligned}
& \quad\left[\frac{1}{h^{2}}-\frac{1}{12}\left(C_{i j}+h D_{x} C_{i j}\right)\right] U_{i-1, j}+ \\
& +\left[\frac{1}{k^{2}}-\frac{1}{12}\left(C_{i j}+k D_{y} C_{i j}\right)\right] U_{i, j-1}+ \\
& +\left(\frac{4}{3} C_{i j}-\frac{2}{h^{2}}-\frac{2}{k^{2}}+\frac{h^{2}}{12} D_{x}^{2} C_{i j}+\frac{k^{2}}{12} D_{y}^{2} C_{i j}\right) U_{i j}+ \\
& +\left[\frac{1}{k^{2}}-\frac{1}{12}\left(C_{i j}-k D_{y} C_{i j}\right)\right] U_{i, j+1}+ \\
& +\left[\frac{1}{h^{2}}-\frac{1}{12}\left(C_{i j}-h D_{x} C_{i j}\right)\right] U_{i+1, j}= \\
& =-F_{i j}-\frac{h^{2}}{12} \Psi\left(X_{i}+[-h, h], Y_{j}\right)-\frac{k^{2}}{12} \Omega\left(X_{i}, Y_{j}+[-h, h]\right)+ \\
& -\frac{h^{2}+k^{2}}{12}[-M, M]+[-\delta, \delta], \quad \\
& \quad i=1,2, \ldots, n-1, \quad j=1,2, \ldots, m-1,
\end{aligned}
$$

while for (26) we obtain

$$
\begin{gathered}
\left(\frac{1}{h^{2}}-\frac{h}{12} D_{x} C_{i j}\right) U_{i-1, j}+\left(\frac{1}{h^{2}}-\frac{h}{12} D_{y} C_{i j}\right) U_{i, j-1}+ \\
+\left[C_{i j}\left(1-\frac{h^{2}}{12} C_{i j}\right)+\frac{h^{2}}{12}\left(D_{x}^{2} C_{i j}+D_{y}^{2} C_{i j}\right)-\frac{4}{h^{2}}\right] U_{i j}+ \\
+\left(\frac{1}{h^{2}}+\frac{h}{12} D_{y} C_{i j}\right) U_{i, j+1}+\left(\frac{1}{h^{2}}+\frac{h}{12} D_{x} C_{i j}\right) U_{i+1, j}= \\
=\left(\frac{h^{2}}{12} C_{i j}-1\right) F_{i j}-\frac{h^{2}}{12}\left\{\Psi\left(X_{i}+[-h, h], Y_{j}\right)+\right. \\
\left.+\Omega\left(X_{i}, Y_{j}+[-h, h]\right)+2[-M, M]\right\}+[-\delta, \delta], \\
i, j=1,2, \ldots, n-1,
\end{gathered}
$$


where $U_{i j}=U\left(X_{i}, Y_{j}\right), F_{i j}=F\left(X_{i}, Y_{j}\right)$, etc., and where the interval $[-\delta, \delta]$ stays for an $\delta$-extension in our methods. In equations (27) this interval contains $O\left(h^{3}\right)+O\left(k^{3}\right)$, and in (28) - the term $O\left(h^{3}\right)$. Moreover, we have

$$
\begin{array}{ll}
U_{0 j}=G_{1}\left(Y_{j}\right), & U_{i 0}=G_{2}\left(X_{i}\right), \\
U_{n j}=G_{3}\left(Y_{j}\right), & U_{i m}=G_{4}\left(X_{i}\right),
\end{array}
$$

for each $j=0,1, \ldots, m$ and $i=0,1, \ldots, n-1$,

where $G_{1}(Y), G_{2}(X), G_{3}(Y)$ and $G_{4}(X)$ denote interval extensions of the functions $g_{1}(y), g_{2}(x), g_{3}(y)$ and $g_{4}(x)$, respectively. The system of linear interval equations (27) with (29) and the system (28) with (29) can be solved in conventional (proper) floating-point interval arithmetic, because all intervals are proper.

Note that if $c(x, y)=c=$ const, then the equations (27) and (28) reduce to

$$
\begin{aligned}
& \left(\frac{1}{h^{2}}-\frac{C}{12}\right) U_{i-1, j}+\left(\frac{1}{k^{2}}-\frac{C}{12}\right) U_{i-1, j}+\left(\frac{4 C}{3}-\frac{2}{h^{2}}-\frac{2}{k^{2}}\right) U_{i j}+ \\
& +\left(\frac{1}{k^{2}}-\frac{C}{12}\right) U_{i, j+1}+\left(\frac{1}{h^{2}}-\frac{C}{12}\right) U_{i+1, j}= \\
& =-F_{i j}-\frac{h^{2}}{12} \Psi\left(X_{i}+[-h, h], Y_{j}\right)-\frac{k^{2}}{12} \Omega\left(X_{i}, Y_{j}+[-h, h]\right)+ \\
& -\frac{h^{2}+k^{2}}{12}[-M, M]+[-\delta, \delta], \quad \\
& i=1,2, \ldots, n-1, \quad j=1,2, \ldots, m-1,
\end{aligned}
$$

and

$$
\begin{aligned}
& \frac{1}{h^{2}} U_{i-1, j}+\frac{1}{h^{2}} U_{i, j-1}+ \\
& +\left[C\left(1-\frac{h^{2}}{12} C\right)-\frac{4}{h^{2}}\right] U_{i j}+\frac{1}{h^{2}} U_{i, j+1}+\frac{1}{h^{2}} U_{i+1, j}= \\
& =\left(\frac{h^{2}}{12} C-1\right) F_{i j}-\frac{h^{2}}{12}\left\{\Psi\left(X_{i}+[-h, h], Y_{j}\right)+\right. \\
& \left.+\Omega\left(X_{i}, Y_{j}+[-h, h]\right)+2[-M, M]\right\}+[-\delta, \delta], \\
& i, j=1,2, \ldots, n-1,
\end{aligned}
$$

respectively, where $C$ is the thinnest machine interval representing $c$. These equations are also useful for solving in interval arithmetic the Poisson equation, in which $c=0$ (see [1115]). Let us recall that Nakao's method is not applicable to the Poisson equation (see Sec. III.).

A remark should be added concerning the constant $M$. When nothing can be concluded about $M$ from physical or technical properties or characteristics of the problem considered, we propose to find this constant taking into account that

$$
\begin{aligned}
& \frac{\partial^{4} u}{\partial x^{2} \partial y^{2}}\left(x_{i}, y_{j}\right)= \\
& =\lim _{h \rightarrow 0} \lim _{k \rightarrow \infty}\left(\frac{u_{i-1, j-1}+u_{i-1, j+1}+u_{i+1, j-1}+u_{i+1, j+1}}{h^{2} k^{2}}+\right. \\
& \left.\quad+\frac{4 u_{i j}-2\left(u_{i-1, j}+u_{i, j-1}+u_{i, j+1}+u_{i+1, j}\right)}{h^{2} k^{2}}\right) .
\end{aligned}
$$

We can calculate

$$
\begin{aligned}
& M_{n m}=\frac{1}{h^{2} k^{2}} \max _{\substack{i=1,2, \ldots, n-1 \\
j=1,2, \ldots, m-1}} \mid u_{i-1, j-1}+u_{i-1, j+1}+u_{i+1, j-1}+ \\
&+u_{i+1, j+1}+4 u_{i j}+ \\
&-2\left(u_{i-1, j}+u_{i, j-1}+u_{i, j+1}+u_{i+1, j}\right) \mid,
\end{aligned}
$$

where $u_{i j}$ are obtained by a conventional method for a variety of $n$ and $m$, say $n=10,20, \ldots, \nu, m=10,20, \ldots, \mu$, where $\nu$ and $\mu$ are sufficiently large. Then, we can plot $M_{n m}$ against different $n$ and $m$. The constant $M$ can be easily determined from the obtained graph, since

$$
\lim _{\substack{n \rightarrow \infty \\ m \rightarrow \infty}} M_{n m} \leq M
$$

For the system of linear equations (27) (and also for (28), (30) and (31)) we can quote the following theorem (see, e.g., [17, p. 89]): If we can carry out all steps of a direct method for solving a finite system of linear algebraic equations $A x=b$ in interval arithmetic (if no attempted division by an interval containing zero occurs, nor any overflow or underflow), then this system has a unique solution for every real matrix in $A$ and every real vector in $b$, and the solution is contained in the resulting interval vector $X$. Because in our method the constant $M$ and $\delta$-extension are obtained experimentally in general, then, obviously, this theorem does not guarantee that we receive validated solution. But all computational experiments that we carried, show that the exact solutions are contained in the interval enclosures obtained by the proposed method (some examples are given in the next section).

\section{A COMPARISON OF NAKAO'S METHOD AND THE INTERVAL DIFFERENCE SCHEME}

First, in this section we compare our method to Nakao's method on the basis of two examples presented by Nakao in [6]. For the third example Nakao's method is not applicable. In all these examples we have used our own implementation of floating-point interval arithmetic written in Delphi Pascal. This implementation has been written in the form of a unit called IntervalArithmetic32and64, whose current version one can find in [16]. This unit takes advantage of the Delphi Pascal floating-point Extended type. All programs written in Delphi Pascal for the examples considered can be found in [18].

Example 1. [6, pp. 330-331]

Let us consider the following problem:

$$
\begin{gathered}
\Delta u+\pi u=(1-2 \pi) \sin \pi x \sin \pi y \text { in } \Omega, \\
u=0 \text { on } \partial \Omega,
\end{gathered}
$$


Tab. 1. Enclosures of solution (34) to the problem (33) obtained by Nakao's method ( $\mathrm{N}$ - intervals presented in [6])




Tab. 2. Enclosures of solution (34) to the problem (33) obtained by the method (31)

\begin{tabular}{|c|c|c|}
\hline$(i, j)$ & $U\left(X_{i}, Y_{j}\right)$ & Width $\times 10^{3}$ \\
\hline$(1,1)$ & $\begin{aligned} {[2.8413489793818334 \mathrm{E}-0002,3.2077265656902749 \mathrm{E}-0002] } & \\
& \approx[0.0284134,0.0320773] \\
\text { exact } & \approx 0.0303959\end{aligned}$ & $\approx 3.7$ \\
\hline$(1,5)$ & $\begin{array}{l}{[9.3435235810096799 \mathrm{E}-0002,1.0258291837474664 \mathrm{E}-0001]} \\
\quad \approx[0.0934352,0.1025830] \\
\text { exact } \approx 0.0983632\end{array}$ & $\approx 9.1$ \\
\hline$(1,9)$ & $\begin{aligned} {[2.8373268255628631 \mathrm{E}-0002,3.2303880411274499 \mathrm{E}-0002] } & \\
& \approx[0.0283732,0.0323039] \\
\text { exact } & \approx 0.0303959\end{aligned}$ & $\approx 3.9$ \\
\hline$(2,1)$ & $\begin{aligned} {[5.4472248034826312 \mathrm{E}-0002,6.0608703745006735 \mathrm{E}-0002] } & \\
& \approx[0.0544722,0.0606088] \\
\text { exact } & \approx 0.0578164\end{aligned}$ & $\approx 6.1$ \\
\hline$(2,5)$ & $\begin{aligned} {[1.7840742966978266 \mathrm{E}-0001,1.9450823450046243 \mathrm{E}-0001] } & \\
& \approx[0.1784074,0.1945083] \\
\text { exact } & \approx 0.1870979\end{aligned}$ & $\approx 16.1$ \\
\hline$(2,9)$ & $\begin{aligned} {[5.4395742122844850 \mathrm{E}-0002,6.1039750622674490 \mathrm{E}-0002] } & \\
& \approx[0.0543957,0.0610398] \\
\text { exact } & \approx 0.0578164\end{aligned}$ & $\approx 6.6$ \\
\hline$(3,1)$ & $\begin{aligned} {[} & 7.5335356959410713 \mathrm{E}-0002,8.3113638230475435 \mathrm{E}-0002] \\
& \approx[0.0753353,0.0831137] \\
\text { exact } & \approx 0.0795775\end{aligned}$ & $\approx 7.8$ \\
\hline$(3,5)$ & 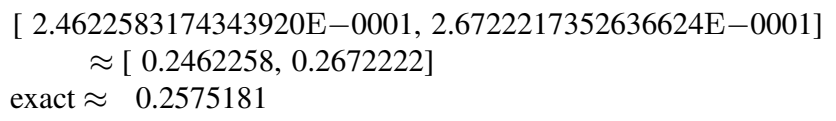 & $\approx 21.0$ \\
\hline$(3,9)$ & 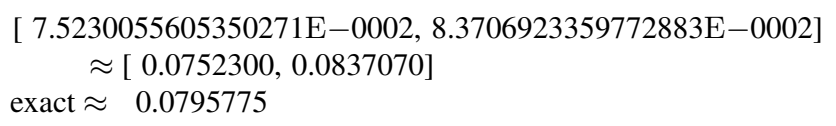 & $\approx 8.5$ \\
\hline$(5,1)$ & $\begin{array}{l}{[9.3435235810096800 \mathrm{E}-0002,1.0258291837474664 \mathrm{E}-0001]} \\
\quad \approx[0.0934352,0.1025830] \\
\text { exact } \approx 0.0983632\end{array}$ & $\approx 9.1$ \\
\hline$(5,5)$ & $\begin{array}{l}{[3.0498232654819171 \mathrm{E}-0001,3.3020640188360169 \mathrm{E}-0001]} \\
\quad \approx[0.3049823,0.3302065] \\
\text { exact } \approx 0.3183099\end{array}$ & $\approx 25.2$ \\
\hline$(5,9)$ & 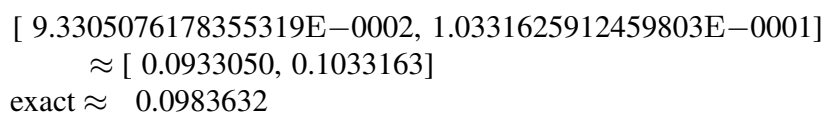 & $\approx 10.0$ \\
\hline$(9,1)$ & 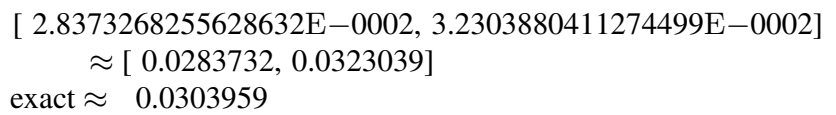 & $\approx 3.9$ \\
\hline$(9,5)$ & 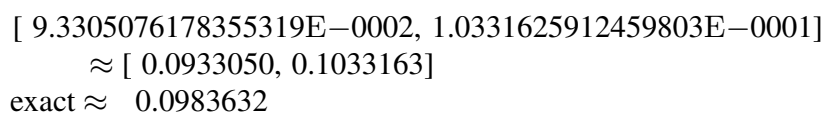 & $\approx 10.0$ \\
\hline$(9,9)$ & $\begin{aligned} {[2.8333046717438929 \mathrm{E}-0002,3.2530495165646248 \mathrm{E}-0002] } & \\
& \approx[0.0283330,0.0325305] \\
\text { exact } & \approx 0.0303959\end{aligned}$ & $\approx 4.2$ \\
\hline
\end{tabular}

where $\Omega=[0,1] \times[0,1]$. The exact solution of (33) is of the form (see Fig. 1)

$$
u(x, y)=\frac{1}{\pi} \sin \pi x \sin \pi y .
$$

For Nakao's method all integrals (8)-(15) can be found analytically. We have

$$
\begin{aligned}
f_{i j}= & \frac{1-2 \pi}{\pi^{2}}(1-\cos \pi h)(\cos \pi(i+j) h+ \\
& \left.-\frac{\sin \pi h}{\pi h} \cos \pi(i-j) h\right), \\
& I_{i j 1}^{c}=\frac{\pi h^{2}}{2}, \quad I_{i j p}^{c}=\frac{\pi h^{2}}{12}, \quad p=2,3, \ldots, 7 .
\end{aligned}
$$


The coefficients $\beta_{i j}$ given by (17) are as follows:

$$
\begin{aligned}
\beta_{i j}= & \frac{\pi h^{2}}{2}\left(u_{i j}^{(k-1)}\right)^{2}+\frac{(2 \pi-1)^{2}}{4}+ \\
& +\left(\frac{2}{\pi}-4\right)(1-\cos \pi h)(\cos \pi(i+j) h+ \\
& \left.-\frac{\sin \pi h}{\pi h} \cos \pi(i-j) h\right) u_{i j}^{(k-1)}
\end{aligned}
$$

Using these formulas in Nakao's method and taking (as Nakao) $h=0.1$, i.e., $n=10$, the initial values $u_{i j}^{(0)}$ from Galerkin's approximation, $\alpha_{i j}^{(0)}=0$, the stopping and extension parameters $\varepsilon=\delta=10^{-8}$, we obtain after $N=10$ iterations the results presented in Tab. 1. In the same table we also present the results obtained by Nakao. One can observe that our intervals are thinner than those presented by Nakao in his original paper. Moreover, it should be added that Nakao obtained his results after $N=16$ iterations, i.e., in a greater number of iterations than in our implementation.

Our interval difference method (31) yields intervals presented in Tab. 2. Since the exact solution is known (see (34), we have taken $M=62.02$. Moreover, we assume in our method that $\delta=0.001$ and, obviously, $U_{0 j}=U_{i 0}=$ $=U_{10, j}=U_{i, 10}=0(j=0,1, \ldots, 10 ; i=1,2, \ldots, 9)$. The obtained enclosures of the exact solution are better than those presented in Tab. 1. Obviously, all intervals (in Tab. 1 and 2) contain the exact solution at the mesh points.

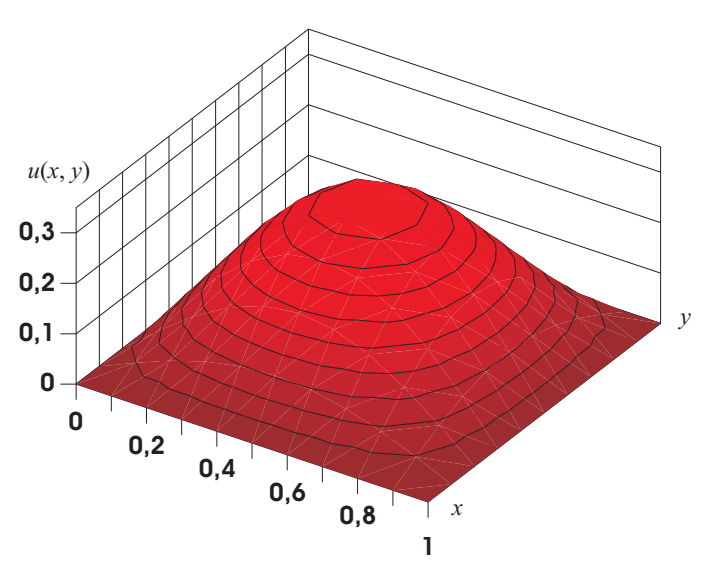

Fig. 1. A graph of solution (34) to the problem (33)

Example 2. [6, pp. 329-330]

For the problem

$$
\begin{gathered}
\Delta u+20 x y u=(1-2 \pi) \sin \pi x \sin \pi y \text { in } \Omega, \\
u=0 \text { on } \partial \Omega,
\end{gathered}
$$

where $\Omega=[0,1] \times[0,1]$, the exact solution is unknown.

Analytical forms of integrals (9)-(15) occurring in Nakao's methods are as follows (the integral (8) is given by (35)):

$$
\begin{aligned}
& I_{i j 1}^{c}=20 h^{4}\left(\frac{1}{2} i j-\frac{1}{36}\right), \\
& I_{i j 2}^{c}=20 h^{4}\left(\frac{1}{12} i j-\frac{1}{24} i-\frac{1}{360}\right), \\
& I_{i j 3}^{c}=20 h^{4}\left(\frac{1}{12} i j+\frac{1}{24} i-\frac{1}{360}\right), \\
& I_{i j 4}^{c}=20 h^{4}\left(\frac{1}{12} i j-\frac{1}{24} j-\frac{1}{360}\right), \\
& I_{i j 5}^{c}=20 h^{4}\left(\frac{1}{12} i j+\frac{1}{24} j-\frac{1}{360}\right), \\
& I_{i j 6}^{c}=20 h^{4}\left(\frac{1}{12} i j+\frac{1}{24} i-\frac{1}{24} j-\frac{1}{45}\right), \\
& I_{i j 7}^{c}=20 h^{4}\left(\frac{1}{12} i j-\frac{1}{24} i+\frac{1}{24} j-\frac{1}{45}\right),
\end{aligned}
$$

while for the components of $\beta_{i j}$ in formula (17) we have

$$
\begin{aligned}
\beta_{i j}^{(1)}= & 400\left(u_{i j}^{(k-1)}\right) h^{6}\left(-\frac{1}{2} i^{3} j^{2}-\frac{1}{2} i^{2} j^{3}-\frac{1}{3} i^{3} j-\frac{1}{6} i^{2} j^{2}+\right. \\
& \quad-\frac{1}{3} i j^{3}-\frac{1}{12} i^{3}-\frac{1}{4} i^{2} j-\frac{1}{4} i j^{2}-\frac{1}{12} j^{3}-\frac{1}{45} i^{2}+ \\
& \left.\quad-\frac{1}{15} i j-\frac{1}{45} j^{2}-\frac{1}{180} i-\frac{1}{180} j+\frac{1}{120}\right) \\
\beta_{i j}^{(2)}= & \frac{20}{\pi^{2}}(2 \pi-1) u_{i j}^{(k-1)} \times \\
\times & \left(\frac { 1 } { 2 \pi } \left\{(i-j)\left[h(\cos 2 \pi h+\cos \pi h)-\frac{3}{2 \pi} \sin 2 \pi h\right]+\right.\right. \\
+ & \left.\frac{1}{\pi}(3 i-7 j+4)\right\} \sin \pi(i-j) h+ \\
+ & \frac{1}{2 \pi}\left[\left(i j h+\frac{5}{2 \pi^{2} h}\right)(2 \sin \pi h-\sin 2 \pi h)+h \sin 2 \pi h+\right. \\
+ & \left.\frac{1}{\pi}(3 \cos 2 \pi h-\cos \pi h)\right] \cos \pi(i-j) h+ \\
+ & \left.\frac{2}{\pi}\left[(i-j) \sin \pi j h-\frac{1}{\pi} \cos \pi j h\right] \cos \pi h \cos \pi i h\right) \\
+ & =\left\{\frac{1}{\pi}\left(\frac{1}{\pi}-h \sin \pi h\right)+\right.
\end{aligned}
$$

and

$$
\int_{0}^{1} \int_{0}^{1} f^{2}(x, y) d x d y=\frac{(2 \pi-1)^{2}}{4} .
$$


Tab. 3. Enclosures of solution to the problem (36) obtained by Nakao's method ( $\mathrm{N}$ - intervals presented in [6])

\begin{tabular}{|c|c|c|}
\hline$(i, j)$ & $U\left(X_{i}, Y_{j}\right)$ & Width $\times 10^{3}$ \\
\hline$(1,1)$ & $\begin{array}{l}\text { [ 2.5422096920147401E-0002, 3.3990702176130446E-0002] } \\
\quad \approx[0.0254220,0.0339908] \\
\mathrm{N} \quad[0.0044953,0.0603775]\end{array}$ & $\begin{array}{l}\approx 8.6 \\
\approx 55.9\end{array}$ \\
\hline$(1,5)$ & $\begin{array}{l}\text { [ } 9.2513778055918294 \mathrm{E}-0002,1.1033908384199032 \mathrm{E}-0001] \\
\quad \approx[0.0925137,0.1103391] \\
\mathrm{N} \quad[0.0066034,0.2148457]\end{array}$ & $\begin{array}{l}\approx 17.8 \\
\approx 208.2\end{array}$ \\
\hline$(1,9)$ & $\begin{array}{l}\text { [ 2.8693572427687149E-0002, 3.7601391162418901E-0002] } \\
\quad \approx[0.0286935,0.0376014] \\
\mathrm{N} \quad[-0.0158443,0.0880232]\end{array}$ & $\begin{array}{c}\approx 8.9 \\
\approx 103.9\end{array}$ \\
\hline$(2,1)$ & $\begin{array}{l}\text { [ 5.0699038788017661E-0002, 6.3495793925095351E-0002] } \\
\quad \approx[0.0506990,0.0634958] \\
\mathrm{N} \quad[0.0097055,0.1147773]\end{array}$ & $\begin{array}{l}\approx 12.8 \\
\approx 105.1\end{array}$ \\
\hline$(2,5)$ & $\begin{array}{l}\text { [ } 1.8060879979601782 \mathrm{E}-0001,2.1053874248141714 \mathrm{E}-0001] \\
\mathrm{N} \quad[\quad[0.1806087,0.2105388] \\
\mathrm{N} \quad 0.0133514,0.4145900]\end{array}$ & $\begin{array}{l}\approx 29.9 \\
\approx 401.2\end{array}$ \\
\hline$(2,9)$ & $\begin{array}{l}\text { [ 5.7342513568695084E-0002, 7.0907900667225485E-0002] } \\
\quad \approx[0.0573425,0.0709080] \\
\mathrm{N} \quad[-0.0269411,0.1674148]\end{array}$ & $\begin{array}{l}\approx 13.6 \\
\approx 194.4\end{array}$ \\
\hline$(3,1)$ & $\begin{array}{l}\text { [ 7.1830592564795797E-0002, 8.7386541698192201E-0002] } \\
\quad \approx[0.0718305,0.0873866] \\
\mathrm{N} \quad[0.0124607,0.1610751]\end{array}$ & $\begin{array}{l}\approx 15.5 \\
\approx 148.6\end{array}$ \\
\hline$(3,5)$ & $\begin{array}{l}\text { [ 2.5492779458340919E-0001, 2.9379815695755907E-0001] } \\
\mathrm{N} \quad \approx \quad[0.2549277,0.2937982] \\
\text { not presented }\end{array}$ & $\approx 38.9$ \\
\hline$(3,9)$ & $\begin{array}{l}\text { [ } 8.2062195994898807 \mathrm{E}-0002,9.8929688472366392 \mathrm{E}-0002] \\
\mathrm{N} \quad \approx[0.0820621,0.0989297] \\
\text { not presented }\end{array}$ & $\approx 16.9$ \\
\hline$(5,1)$ & $\begin{array}{l}\text { [ } 9.2513778055918294 \mathrm{E}-0002,1.1033908384199032 \mathrm{E}-0001] \\
\mathrm{N} \quad \approx[0.0925137,0.1103391] \\
\quad \text { not presented }\end{array}$ & $\approx 17.8$ \\
\hline$(5,5)$ & $\begin{array}{l}\text { [ } 3.3138320035174136 \mathrm{E}-0001,3.7902599829881106 \mathrm{E}-0001] \\
\mathrm{N} \quad \text { [ } 0.3313832,0.3790260] \\
\text { not presented }\end{array}$ & $\approx 47.6$ \\
\hline$(5,9)$ & $\begin{array}{l}\text { [ } 1.0874662190671927 \mathrm{E}-0001,1.2906568116001579 \mathrm{E}-0001] \\
\mathrm{N} \quad \approx[0.1087466,0.1290657] \\
\text { not presented }\end{array}$ & $\approx 20.3$ \\
\hline$(9,1)$ & $\begin{array}{l}\text { [ 2.8693572427687149E-0002, 3.7601391162418901E-0002] } \\
\mathrm{N} \quad \approx[0.0286935,0.0376014] \\
\text { not presented }\end{array}$ & $\approx 8.9$ \\
\hline$(9,5)$ & $\begin{array}{l}\text { [ } 1.0874662190671927 \mathrm{E}-0001,1.2906568116001579 \mathrm{E}-0001] \\
\mathrm{N} \quad \begin{array}{l}\text { [ } 0.1087466,0.1290657] \\
\text { not presented }\end{array}\end{array}$ & $\approx 20.3$ \\
\hline$(9,9)$ & $\begin{array}{l}\text { [ } 3.5234034840690512 \mathrm{E}-0002,4.5480438346456136 \mathrm{E}-0002] \\
\mathrm{N} \quad \approx[0.0352340,0.0454805] \\
\text { not presented }\end{array}$ & $\approx 10.2$ \\
\hline
\end{tabular}

Taking (as Nakao) $h=0.1$, the initial values $u_{i j}^{(0)}$ from the Galerkin approximation, $\alpha_{i j}^{(0)}=0$, the stopping parameter $\varepsilon=10^{-4}$ and the extension parameter $\delta=10^{-3}$, in our implementation of Nakao's method we obtain after $N=7$ iterations the results presented in Tab. 3. The Nakao results (published in [6]) obtained after $N=10$ are also presented in the same table. As in Example 1 we can observe that our intervals are significant thinner and Nakao obtained his results after a greater number of iterations.

In order to use the interval difference method (28) we need to evaluate the constant $M$. Since the exact solution is unknown, we can calculate $M_{n m}$ from (32) for different values of $n$. The obtained results are presented in Fig. 2. From this figure it follows that $M=38.9$. For $h=0.1, \delta=0.001$ 
Tab. 4. Enclosures of solution to the problem (36) obtained by the method (28)

\begin{tabular}{|c|c|c|}
\hline$(i, j)$ & $U\left(X_{i}, Y_{j}\right)$ & Width $\times 10^{3}$ \\
\hline$(1,1)$ & 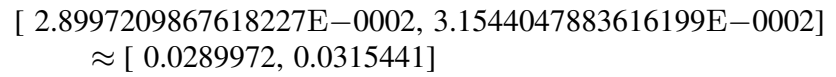 & $\approx 2.5$ \\
\hline$(1,5)$ &  & $\approx 6.9$ \\
\hline$(1,9)$ & $\begin{array}{l}{[3.1726223260720911 \mathrm{E}-0002,3.4740582521688013 \mathrm{E}-0002]} \\
\quad \approx[0.0317262,0.0347406]\end{array}$ & $\approx 3.0$ \\
\hline$(2,1)$ & $\begin{array}{l}{[5.5847687322568013 \mathrm{E}-0002,6.0189730374459988 \mathrm{E}-0002]} \\
\quad \approx[0.0558476,0.0601898]\end{array}$ & $\approx 4.3$ \\
\hline$(2,5)$ & $\begin{array}{l}{[1.9176543915299421 \mathrm{E}-0001,2.0413632819753807 \mathrm{E}-0001]} \\
\quad \approx[0.1917654,0.2041364]\end{array}$ & $\approx 12.4$ \\
\hline$(2,9)$ & $\begin{array}{l}{[6.1877653239000653 \mathrm{E}-0002,6.7170747737222414 \mathrm{E}-0002]} \\
\quad \approx[0.0618776,0.0671708]\end{array}$ & $\approx 5.3$ \\
\hline$(3,1)$ &  & $\approx 5.6$ \\
\hline$(3,5)$ & $\begin{array}{l}{[2.6958027386866717 \mathrm{E}-0001,2.8619531804216802 \mathrm{E}-0001]} \\
\quad \approx[0.2695802,0.2861954]\end{array}$ & $\approx 16.6$ \\
\hline$(3,9)$ & $\begin{array}{l}{[8.7847100544052620 \mathrm{E}-0002,9.4883951515621320 \mathrm{E}-0002]} \\
\quad \approx[0.0878471,0.0948840]\end{array}$ & $\approx 7.0$ \\
\hline$(5,1)$ & 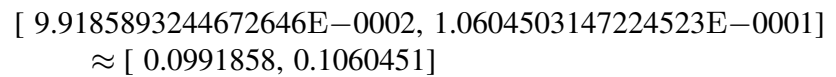 & $\approx 6.9$ \\
\hline$(5,5)$ & $\begin{array}{l}{[3.4971929521257995 \mathrm{E}-0001,3.7100087427077752 \mathrm{E}-0001]} \\
\quad \approx[0.3497192,0.3710009]\end{array}$ & $\approx 21.3$ \\
\hline$(5,9)$ & $\begin{array}{l}{[1.1638954152907525 \mathrm{E}-0001,1.2539898721724767 \mathrm{E}-0001]} \\
\quad \approx[0.1163895,0.1253990]\end{array}$ & $\approx 9.0$ \\
\hline$(9,1)$ & $\begin{array}{l}{[3.1726223260720911 \mathrm{E}-0002,3.4740582521688013 \mathrm{E}-0002]} \\
\quad \approx[0.0317262,0.0347406]\end{array}$ & $\approx 3.0$ \\
\hline$(9,5)$ & $\begin{array}{l}{[1.1638954152907525 \mathrm{E}-0001,1.2539898721724767 \mathrm{E}-0001]} \\
\quad \approx[0.1163895,0.1253990]\end{array}$ & $\approx 9.0$ \\
\hline$(9,9)$ & $\begin{array}{l}{[4.0001555361422584 \mathrm{E}-0002,4.3990214693613634 \mathrm{E}-0002]} \\
\quad \approx[0.0400015,0.0439903]\end{array}$ & $\approx 4.0$ \\
\hline
\end{tabular}

and $U_{0 j}=U_{i 0}=U_{10, j}=U_{i, 10}=0(j=0,1, \ldots, 10 ; i=$ $=1,2, \ldots, 9)$, from $(28)$ we obtain enclosures of the exact solution presented in Tab. 4 (these enclosure are thinner than those presented in Tab. 3).

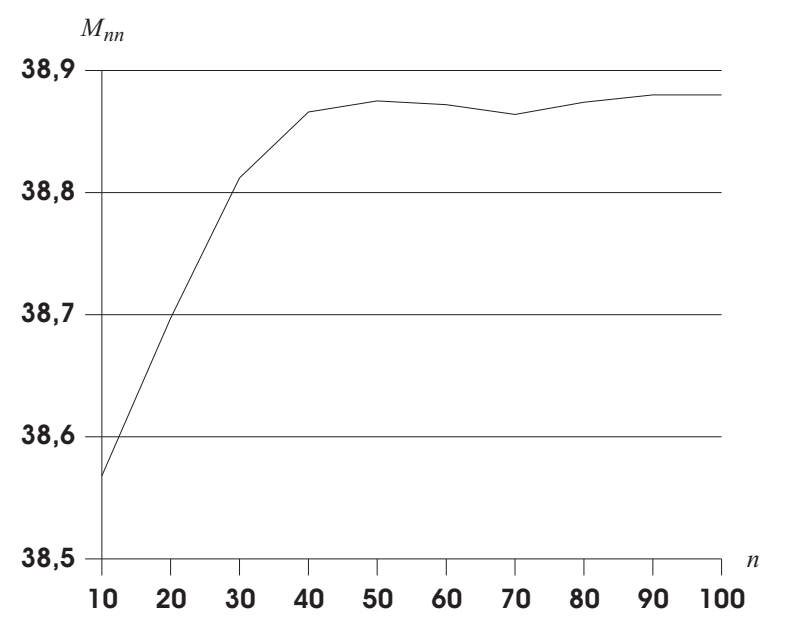

Fig. 2. A graph for $M_{n n}$ obtained from (32) for $n=10,20, \ldots, 100$

\section{Example 3. Let}

$$
\begin{aligned}
\Delta u= & \frac{1}{1+x^{2}+y^{2}}\left[\frac{2+x^{2}+y^{2}}{\sqrt{1+x^{2}+y^{2}}} \cos \sqrt{1+x^{2}+y^{2}}+\right. \\
& \left.-\left(x^{2}+y^{2}\right) \sin \sqrt{1+x^{2}+y^{2}}\right] \text { in } \Omega
\end{aligned}
$$

and

$$
\left.u\right|_{\partial \Omega}=\left\{\begin{array}{l}
\sin \sqrt{1+y^{2}} \text { for } x=0, \\
\sin \sqrt{1+x^{2}} \text { for } y=0 \\
\sin \sqrt{65+y^{2}} \text { for } x=8, \\
\sin \sqrt{17+x^{2}} \text { for } y=4,
\end{array}\right.
$$

where $\Omega=[0,8] \times[0,4]$. The Poisson equation (37) with Dirichlet's conditions (38) has the solution of the form (see Fig. 3)

$$
u(x, y)=\sin \sqrt{1+x^{2}+y^{2}} .
$$


Tab. 5. Enclosures of solution (39) to the problem (37)-(38) obtained by the method (31) (for selected $i$ and $j$ )

\begin{tabular}{|c|c|c|}
\hline$(i, j)$ & $U\left(X_{i}, Y_{j}\right)$ & Width $\times 10^{3}$ \\
\hline$(40,8)$ & $\begin{aligned} {[-8.7570448853762087 \mathrm{E}-0001,-8.6786852449020102 \mathrm{E}-0001] } & \\
& \approx[-0.8757044,-0.8678686] \\
\mathrm{exact} & \approx-0.8715758\end{aligned}$ & $\approx 7.8$ \\
\hline$(40,16)$ & $\begin{aligned} {[-9.6473261912851148 \mathrm{E}-0001,-9.5273425661315874 \mathrm{E}-0001] } & \\
& \approx[-0.9647326,-0.9527343] \\
\mathrm{exact} & \approx-0.9583239\end{aligned}$ & $\approx 12.0$ \\
\hline$(40,24)$ & $\begin{aligned} {[-1.0049018906555416 \mathrm{E}+0000,-9.9269471083206701 \mathrm{E}-0001] } & \\
& \approx[-1.0049018,-0.9926948] \\
\text { exact } & \approx-0.9982978\end{aligned}$ & $\approx 12.2$ \\
\hline$(40,32)$ & $\begin{aligned} {[-8.7882382725746346 \mathrm{E}-0001,-8.7056138147601539 \mathrm{E}-0001] } & \\
& \approx[-0.8788238,-0.8705614] \\
\text { exact } & \approx-0.8742991\end{aligned}$ & $\approx 8.3$ \\
\hline$(16,20)$ & 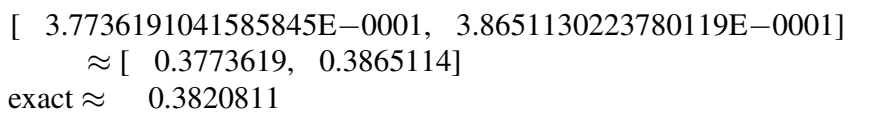 & $\approx 9.1$ \\
\hline$(32,20)$ & $\begin{aligned} {[-6.9697972969609782 \mathrm{E}-0001,-6.8473622032926368 \mathrm{E}-0001] } & \\
& \approx[-0.6969797,-0.6847363] \\
\text { exact } & \approx-0.6905517\end{aligned}$ & $\approx 12.2$ \\
\hline$(48,20)$ & $\begin{aligned} {[-8.4156500228698132 \mathrm{E}-0001,-8.2916731158025701 \mathrm{E}-0001] } & \\
& \approx[-0.8415650,-0.8291674] \\
\text { exact } & \approx-0.8348743\end{aligned}$ & $\approx 12.4$ \\
\hline$(64,20)$ & 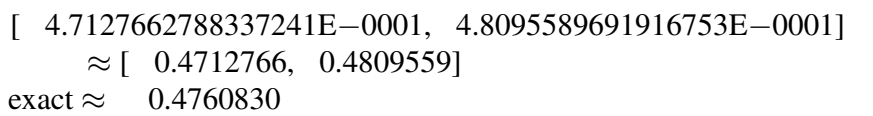 & $\approx 9.7$ \\
\hline
\end{tabular}

The problem (37)-(38) cannot be solved by Nakao's method since $c=0$ (see Sec. III.). But using the method presented in Sec. V. we can obtain enclosures of (39) at some mesh points.

Taking $n=80, m=40(h=k=0.1), M=1.382$ (this constant can be estimated since the exact solution is known) from the method (31), in which $C=0$ and the interval $[-\delta, \delta]$ does not occur (this interval never occurs for the Poisson equation), and for

$$
\begin{gathered}
U_{0 j}=\sin \sqrt{1+Y_{j}^{2}}, \quad U_{i 0}=\sin \sqrt{1+X_{i}^{2}}, \\
U_{80, j}=\sin \sqrt{65+Y_{j}^{2}}, \quad U_{i 0}=\sin \sqrt{17+X_{i}^{2}}, \\
j=0,1, \ldots, 40, \quad i=1,2, \ldots, 79,
\end{gathered}
$$

we have obtained enclosures of the exact solution presented in Tab. 5. As previously, all intervals contain the exact solution at the mesh points.

\section{CONCLUSIONS}

In the paper we have shown that our implementation of Nakao's method yields better enclosures of the exact solutions than those presented in the original paper [6]. There are two possible reasons for that: we have used the exact integrals in the method, while probably in [6] some quadratures have been used (it causes to come into being some additional errors - errors of quadratures), and in our interval calculations we have taken advantage of Delphi Pascal Extended type, which is more precise than Double type used probably in [6] (this has rather small influence on the results).

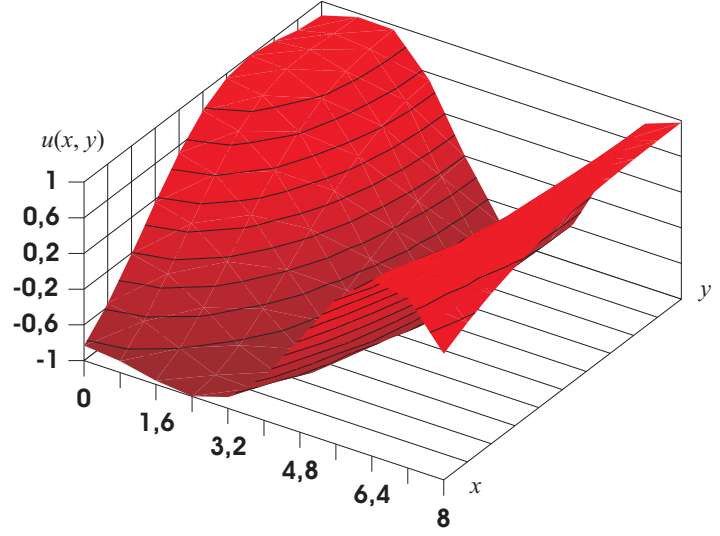

Fig. 3. A graph of solution (39) to the problem (37)-(38)

Although Nakao's method can be applied to a lot of elliptic boundary value problems, the method cannot be applied for $(b, c)=(0,0)$, which means that, e.g., the conventional Poisson equation cannot be solved. Our interval difference scheme is free from this disadvantage. Moreover, our method can be applied for functions $u(x, y) \neq 0$ on the 
boundary $\partial \Omega$, we can use different mesh sizes $h$ and $k$ in $x$ - and $y$-directions, respectively, and the boundary $\partial \Omega$ does not need to be restricted to $[0,1] \times[0,1]$. It should be added that the last remark is not very important, since for an arbitrary boundary $\left[\alpha_{1}, \alpha_{2}\right] \times\left[\beta_{1}, \beta_{2}\right]$ we can always take new variables $s$ and $t$ (instead of $x$ and $y$ ) defined as follows:

$$
s=\frac{x-\alpha_{1}}{\alpha_{2}-\alpha_{1}}, \quad t=\frac{x-\beta_{1}}{\beta_{2}-\beta_{1}},
$$

for which the region $\left[\alpha_{1}, \alpha_{2}\right] \times\left[\beta_{1}, \beta_{2}\right]$ will be transformed to $[0,1] \times[0,1]$.

Finally, we want to draw attention to time of computations. Since Nakao's method is an iterative process, the method is time-expansive and the time of computations grows rapidly when we decrease the mesh size $h$ or/and the iteration accuracy $\varepsilon$. In our method the execution time depends only on the mesh sizes $h$ and $k$ (of course, this time grows for smaller $h$ and $k$ ). In our programs, presented in [18], the execution time is always calculated.

\section{Acknowledgment}

The paper was supported by Poznań University of Technology (Poland) through Grant No. 09/91/DSPB/1649.

\section{References}

[1] R.E. Moore, Interval Analysis, Prentice-Hall, Englewood Cliffs (1966).

[2] R.E. Moore, Methods and Applications of Interval Analysis, SIAM, Philadelphia (1979).

[3] G. Alefeld, J. Herzberger, Introduction to Interval Computations, Academic Press, New York (1983).

[4] R. Hammer, M. Hocks, U. Kulisch, D. Ratz, Numerical Toolbox for Verified Computing I. Basic Numerical Problems, Theory, Algorithms, and Pascal-XSC Programs, SpringerVerlag, Berlin (1993).

[5] E.R. Hansen, Topics in Interval Analysis, Oxford University Press, London (1969).
[6] M.T. Nakao, A Numerical Approach to the Proof of Existence of Solutions for Elliptic Problems, Japan Journal of Applied Mathematics 5, 313-332 (1988).

[7] M.T. Nakao, Solving Nonlinear Elliptic Problems with Result Verification Using H-1 Type Residual Iteration, Computing (Suppl.) 9, 161-173 (1993).

[8] M.T. Nakao, On Verified Computations of Solutions for Nonlinear Parabolic Problems, Nonlinear Theory and Its Applications. IEICE 5(3), 320-338 (2014).

[9] T. Meis, U. Marcowitz, Numerical Solution of Partial Differential Equations, Springer-Verlag, New York (1981).

[10] E. Süli, Lecture Notes on Finite Element Methods for Partial Differential Equations, University of Oxford (2000). https://people.maths.ox.ac.uk/suli/fem.pdf

[11] T. Hoffmann, A. Marciniak, Solving the Generalized Poisson Equation in Proper and Directed Interval Arithmetic, Computational Methods in Science and Technology 22(4), 225232 (2016).

[12] T. Hoffmann, A. Marciniak, B. Szyszka, Interval Versions of Central Difference Method for Solving the Poisson Equation in Proper and Directed Interval Arithmetic, Foundations of Computing and Decision Sciences 38(3), 193-206 (2013).

[13] T. Hoffmann, A. Marciniak, Solving the Poisson Equation by an Interval Method of the Second Order, Computational Methods in Science and Technology 19(1), 13-21 (2013).

[14] A. Marciniak, T. Hoffmann, Interval Difference Methods for Solving the Poisson Equation, [In:] S. Pinelas, T. Caraballo, P. Kloeden, J.R. Graef (eds.), Differential and Difference Equations with Applications, vol. 230 of Springer Proceedings in Mathematics \& Statistics, 259-270, Springer (2018).

[15] A. Marciniak, An Interval Difference Method for Solving the Poisson Equation - the First Approach, Pro Dialog 24, 49-61 (2008).

[16] A. Marciniak, Interval Arithmetic Unit, (2016). http://www.c s.put.poznan.pl/amarciniak/IAUnits/IntervalArithmetic32and 64.pas

[17] R.E. Moore, R.B. Kearfott, M.J. Cloud, Introduction to Interval Analysis, SIAM, Philadelphia (2009).

[18] A. Marciniak, Delphi Pascal Programs for Nakao and Interval Difference Methods for Solving the Elliptic BVP, (2019). http://www.cs.put.poznan.pl/amarciniak/NIDM-EllipticBVP -Examples

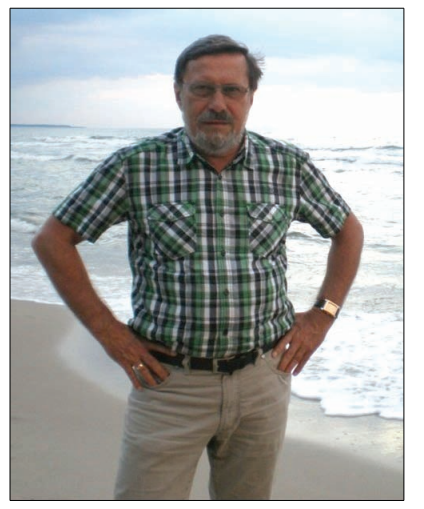

Andrzej Marciniak was born in Poznań (Poland) in 1953. He received the M.Sc. degree in mathematics in 1977, the M.Sc. degree in astronomy in 1979 and Ph.D. degree in mathematics in 1981, all from the Adam Mickiewicz University in Poznań. In 1993 he received the Dr.Habil. degree in physics from the Nicolaus Copernicus University in Torun (Poland) and in 2010 he received the Professor Title from the President of Poland. From 1977 to 1987 and from 2000 to 2011 he held a research position at the Faculty of Mathematics and Computer Science of the Adam Mickiewicz University, and since 1987 he has been an an assistant professor in Institute of Mathematics and then a professor of computer science at the Faculty of Computing Science of the Poznań University of Technology. From 2005 to 2008 he held the office of the President of Polish Information Processing Society. His research interests include computer programming and numerical methods, especially for solving ordinary and partial differential equations with applications to dynamical problems. In these fields he wrote three monographs, more than 20 textbooks and a number of scientific articles. 